\title{
Factor XII and uPAR upregulate neutrophil functions to influence wound healing
}

\author{
Evi X. Stavrou, ${ }^{1,2}$ Chao Fang, ${ }^{2}$ Kara L. Bane, ${ }^{2}$ Andy T. Long, ${ }^{3}$ Clément Naudin, ${ }^{4}$ Erdem Kucukal, ${ }^{5}$ Agharnan Gandhi, ${ }^{2}$ \\ Adina Brett-Morris, ${ }^{2}$ Michele M. Mumaw, ${ }^{2}$ Sudeh Izadmehr, ${ }^{6}$ Alona Merkulova, ${ }^{2}$ Cindy C. Reynolds, ${ }^{2}$ Omar Alhalabi, ${ }^{2}$ \\ Lalitha Nayak, ${ }^{2,7}$ Wen-Mei Yu, ${ }^{2}$ Cheng-Kui Qu, ${ }^{2}$ Howard J. Meyerson, ${ }^{8}$ George R. Dubyak, ${ }^{9}$ Umut A. Gurkan, ${ }^{5}$ \\ Marvin T. Nieman, ${ }^{10}$ Anirban Sen Gupta, ${ }^{11}$ Thomas Renné, ${ }^{3,4}$ and Alvin H. Schmaier ${ }^{2,7}$ \begin{abstract}
School of Medicine, Cleveland, Ohio, USA. ${ }^{3}$ Institute of Clinical Chemistry and Laboratory Medicine, University Medical Center Hamburg-Eppendorf, Hamburg, Germany. ${ }^{4}$ Clinical Chemistry, Department of Sciences, Graduate School of Biomedical Sciences, Icahn School of Medicine at Mount Sinai, New York, New York, USA. 'Department of Medicine, Hematology and Oncology Division, University Hospitals Cleveland Medical Center, Cleveland, Ohio, USA. ${ }^{8}$ Department of Pathology, ${ }^{9}$ Department of Physiology and Biophysics, ${ }^{10}$ Department of Pharmacology, and ${ }^{11}$ Department of Biomedical Engineering,
\end{abstract} \\ 'Department of Medicine, Louis Stokes Veterans Administration Medical Center, Cleveland, Ohio, USA. ²Department of Medicine, Hematology and Oncology Division, Case Western Reserve University (CWRU) \\ Molecular Medicine and Surgery, Karolinska Institutet, Stockholm, Sweden. ${ }^{5}$ Department of Mechanical and Aerospace Engineering, CWRU, Cleveland, Ohio, USA. ${ }^{6}$ Department of Genetics and Genomics \\ CWRU, Cleveland, Ohio, USA.
}

\begin{abstract}
Coagulation factor XII (FXII) deficiency is associated with decreased neutrophil migration, but the mechanisms remain uncharacterized. Here, we examine how FXII contributes to the inflammatory response. In 2 models of sterile inflammation, FXII-deficient mice $\left(F 2^{-/-}\right)$had fewer neutrophils recruited than WT mice. We discovered that neutrophils produced a pool of FXII that is functionally distinct from hepatic-derived FXII and contributes to neutrophil trafficking at sites of inflammation. FXII signals in neutrophils through urokinase plasminogen activator receptor-mediated (uPAR-mediated) Akt2 phosphorylation at $S^{474}$ (pAktS ${ }^{474}$ ). Downstream of pAkt2S ${ }^{474}$, FXII stimulation of neutrophils upregulated surface expression of $\alpha_{M} \beta_{2}$ integrin, increased intracellular calcium, and promoted extracellular DNA release. The sum of these activities contributed to neutrophil cell adhesion, migration, and release of neutrophil extracellular traps in a process called NETosis. Decreased neutrophil signaling in $\mathrm{F}^{-/ /-}$mice resulted in less inflammation and faster wound healing. Targeting hepatic F12 with siRNA did not affect neutrophil migration, whereas WT BM transplanted into F12 ${ }^{-/-}$hosts was sufficient to correct the neutrophil migration defect in $\mathrm{F12}^{-/-}$mice and restore wound inflammation. Importantly, these activities were a zymogen FXII function and independent of FXIla and contact activation, highlighting that FXII has a sophisticated role in vivo that has not been previously appreciated.
\end{abstract}

\section{Introduction}

Factor XII (FXII) is the zymogen of serine protease factor XIIa (FXIIa). Discovered by Ratnoff 60 years ago, FXII was first recognized as essential for surface-activated blood coagulation. FXII is activated to its enzyme by plasma kallikrein or by its unique property of autoactivating from a zymogen to an enzyme (FXIIa) when bound to artificial or certain biologic surfaces (1, 2). The mechanism for autoactivation is not completely characterized. New interest in FXII has been kindled by the recognition that $\mathrm{F}^{-/ /-}$mice have reduced thrombosis, but normal hemostasis, and by the identification of several biologic substances (e.g., polyphosphates, aggregated protein, RNA, vascular collagen, neutrophil extracellular traps [NETs]) that support FXII autoactivation (3-15). Since FXII deficiency is not associated with a defect in hemostasis, inhibition of FXIIa, the enzymatic form of FXII, has emerged as a potential target for preventing thrombosis without increasing bleeding risk.

Conflict of interest: The authors have declared that no conflict of interest exists. Submitted: January 26, 2017; Accepted: December 14, 2017. Reference information: / Clin Invest. 2018;128(3):944-959. https://doi.org/10.1172/JCI92880.
Although FXII enzymatic activities have been extensively studied, few zymogen FXII-initiated functions have been appreciated. Older studies showed that FXII deficiency is associated with decreased migration of inflammatory cells into skin windows (16). In human plasma, FXII and FXIIa induce neutrophil aggregation (17) and plasma FXII is present on the surface of neutrophils (18). Zymogen FXII stimulates monocyte expression of Fc $\gamma$ RII (19). In contrast to other components of the coagulation system, FXII has 2 EGF domains and the zymogen has mitogenic activity in smooth muscle and endothelial cells where FXII stimulates angiogenesis through urokinase plasminogen activator receptor (uPAR) (20-22). Activation of dendritic cells by FXII also modulates adaptive immunity through uPAR-mediated cytokine production, contributing to neuroinflammation (23). These data indicate that zymogen FXII regulates cell biology independently of its protease function.

Here, we examined the role of FXII in the inflammatory response. Immunofluorescence and surface plasmon resonance show that, following neutrophil activation, autocrine FXII binds to uPAR on the neutrophil surface in a zinc-dependent $\left(\mathrm{Zn}^{2+}\right)$ manner. Signaling assays demonstrate that the FXII-uPAR interaction promotes Akt $2 \mathrm{~S}^{474}$ phosphorylation, upregulates the membrane 
A Day 2

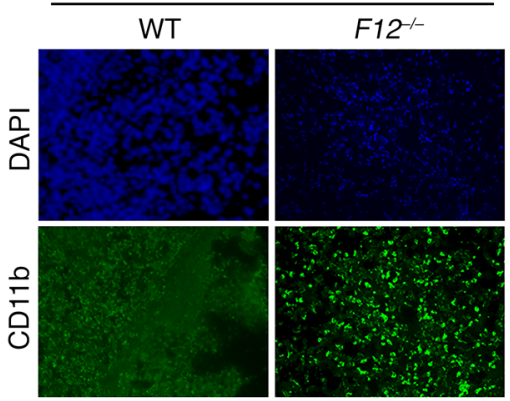

C

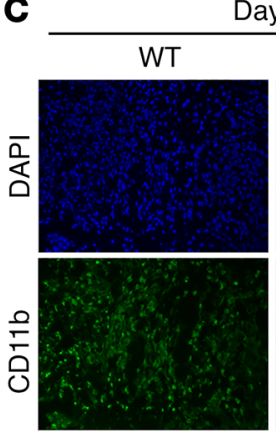

Day 5

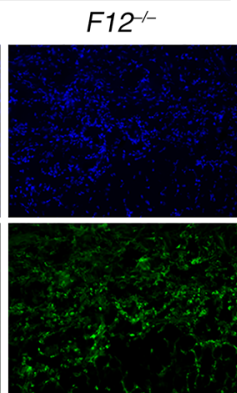

$\mathbf{E}$

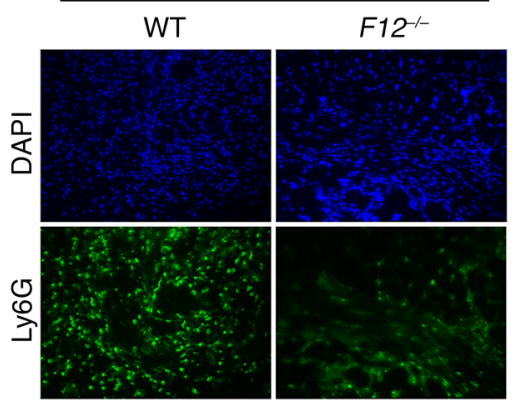

G

Day 5

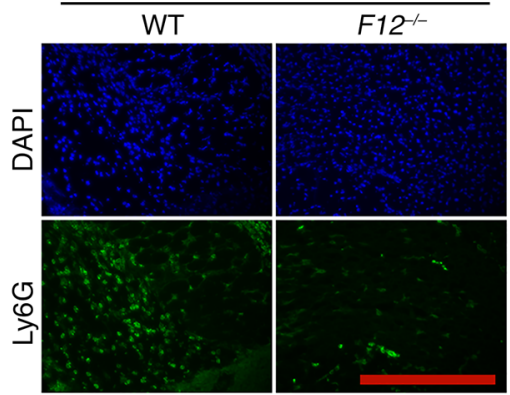

B

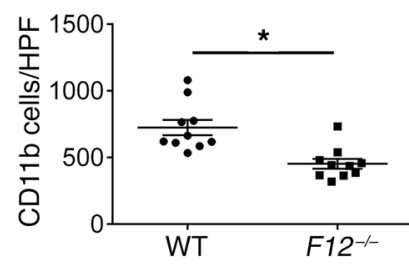

D

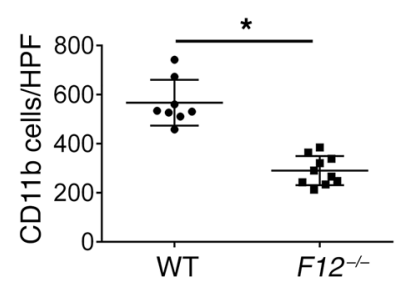

$\mathbf{F}$

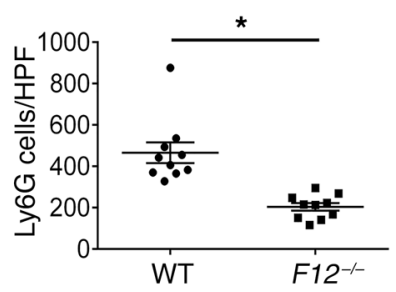

H

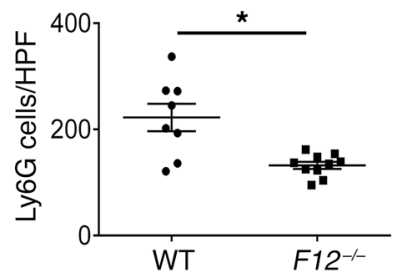

Figure 1. FXII influences leukocyte migration into skin wounds. (A and C) Frozen sections of day 2 (D2) and day 5 (D5) wounds were stained with anti-CD11b antibody to assess leukocyte infiltration. (B and D) Total number of CD11b cells per high-power field (HPF). WT, $n=10 ; F 12^{-/-}, n=10$ mice (B); WT, $n=8 ; F 12^{-/}, n=10$ mice (D). (E and $\mathbf{G})$ Representative frozen sections from day 2 and day 5 wounds were stained with anti-Ly6C antibody to determine neutrophil infiltration. ( $\mathbf{F}$ and $\mathbf{H}$ ) The numbers of Ly6G cells/highpower field are shown. WT, $n=10 ; F 12^{-1-}, n=10$ mice $(\mathbf{F})$; WT, $n=8$; $\mathrm{F}^{2-1-}, n=10$ mice $(\mathrm{H})$. Fluorescent images were obtained using a Nikon TE2000-S microscope at $\times 20$ magnification. CD11b and Ly6C staining in all panels was compared by morphometric analysis of cell number per high-power field using Imagel software (NIH). Data represent mean \pm SEM. ${ }^{*} P<0.01$ vs. WT control mice by Student's $t$ test. Scale bar: $50 \mu \mathrm{m}$

FXII/uPAR axis in neutrophils has the potential to reduce inflammation and promote wound healing.

\section{Results}

Characterization of $\mathrm{F} 12^{-/-}$mice. $\mathrm{F} 12^{-/-}$mice on a C57BL/6J background were generated by NEO insertion in place of $F 12$ exons 3-8 (24). The mice were not the $F 12^{-/-}$mice that had a concurrent profilin deletion (25). Upon PCR of genomic DNA, WT $\left(F 12^{+/+}\right)$and $F 12^{-/-}$genes produced 152bp and 128-bp bands, respectively (Supplemental Figure 1A; supplemental material available online with this article; https://doi.org/10.1172/JCI92880DS1. See complete unedited blots in the supplemental material). FXII-deficient plasma had a significantly prolonged activated partial thromboplastin time (aPTT) (Supplemental Figure 1B). FXII-deficient plasma had less than 5\% FXII coagulant activity compared with normal plasma (Supplemental Figure 1C). Immunoblot studies showed no detectable FXII antigen in FXII-deficient plasma (Supplemental Figure 1D; see complete unedited blots in the supplemental material). F12 ${ }^{-/}$mice exhibited normal hematologic parameters and prothrombin time (Supplemental Table 1).

The influence of FXII in inflammation. Two observations led us to investigate whether FXII participates in the inflammatory response and wound repair. First, $F 12^{-/-}$mice had reduced wound angiogenesis mediated by uPAR, and second, uPAR influenced leukocyte recruitment and inflammation $(22,26)$. Sterile skin punch biopsies $(5 \mathrm{~mm})$ were taken from WT and $\mathrm{F}^{-1 /-}$ mice. Wounds were harvested on days 2 and 5 , and frozen sections were stained with anti-CD11b, a leukocyte marker (Figure 1, A and C). There was an approxtranslocation of $\alpha_{M} \beta_{2}$ integrin, increases intracellular calcium concentration, and leads to extracellular DNA release. Real-time microfluidic visualization of neutrophils shows that FXII is a potent chemotaxin. Neutrophils in FXII-deficient mice exhibit reduced adhesion, migration, and chemotaxis. Moreover, transplantation of BM from FXII-deficient mice into WT hosts results in faster wound healing. Studies with FXII variants confirm that FXII-mediated signaling in neutrophils is independent of its enzymatic activity. Taken together, these findings provide evidence that FXII/uPAR contributes to key neutrophil functions to influence cell trafficking at sites of inflammation. Further, targeting the imately 2-fold decrease in CD11b staining in $\mathrm{F} 12^{-/-}$wounds versus WT on day $2(P=0.0009)$ (Figure $1 \mathrm{~B})$ and day $5(P=0.0001)$ (Figure 1D). Leukocyte subpopulation analysis using anti-Ly6G antibody, a neutrophil marker, also indicated a disproportionate decrease in the neutrophil population that appeared in $\mathrm{F} 12^{-/-}$ wounds on both day $2(P=0.0001)$ (Figure $1, \mathrm{E}$ and $\mathrm{F}$ ) and day 5 $(P=0.0018)$ (Figure $1, \mathrm{G}$ and $\mathrm{H})$.

The absence of FXII is associated with a $50 \%$ reduction in bradykinin (BK) formation (24), and BK itself influences leukocyte function $(27,28)$. To determine whether reduced bradykinin signaling contributed to the findings of decreased leukocyte 

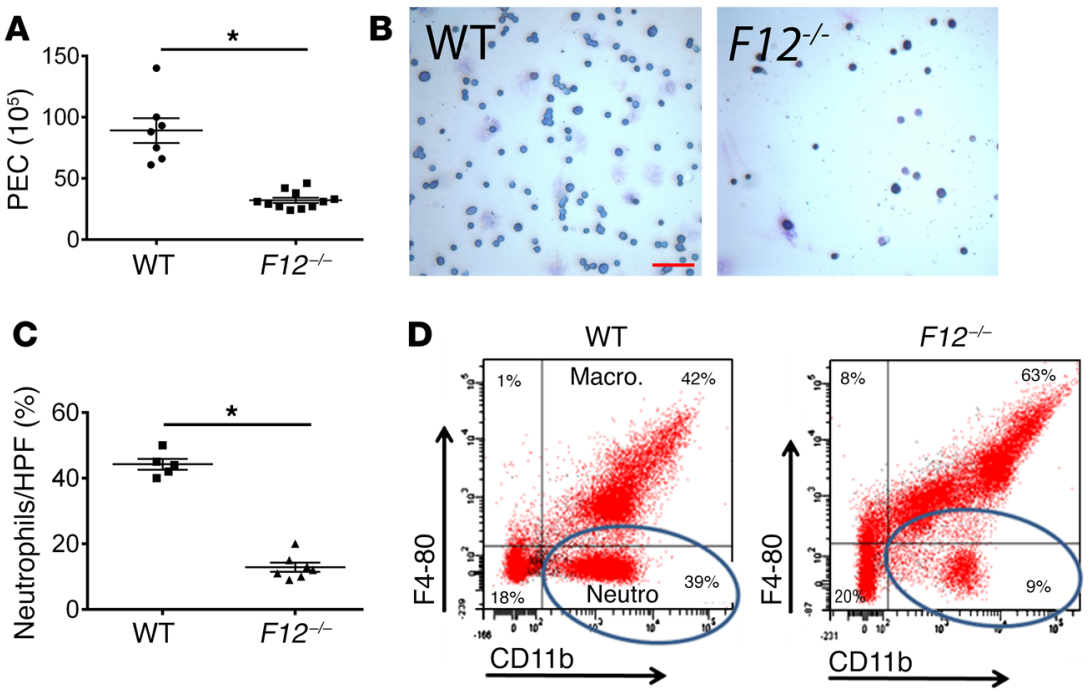

Figure 2. Leukocyte migration in TG-induced peritonitis. WT and $\mathrm{F} 12^{-/-}$mice were injected intraperitoneally with TC solution. At 4 hours (A), mice were subjected to peritoneal lavage and the PEC number was determined. WT, $n=7 ; F 12^{-/-}, n=11$ mice (mean $\left.\pm \mathrm{SEM}\right) .{ }^{*} P$ $=0.0001$ vs. WT control mice by Student's $t$ test. (B) Giemsa-Wright stain of peritoneal lavage fluid. Original magnification, $\times 4$. Scale bar: $10 \mu \mathrm{m}$. Representative images of $n=5$ WT and $n=7$ F12 ${ }^{-/-}$animals. (C) Quantitation of neutrophil population in the peritoneal lavage fluid of WT and $F 12^{-/-}$mice 4 hours after TC instillation as observed by light microscopy (WT, $n=5 ; F 12^{-/-}, n=7$ ). Data represent mean \pm SEM. ${ }^{*} P<0.0001$ vs. WT control mice by Student's $t$ test. (D) Murine peritoneal lavage fluid was labeled with PE-conjugated anti-CD11b and PerCPCy 5.5-conjugated anti-F4-80 antibodies. Circles indicate the neutrophil subpopulation (CD11b-positive, F4-80-negative cells). These figures are representative of flow cytograms of $n=4$ animals in each group. recruitment in skin wounds, we examined BK B2 receptor-deleted $\left(B d k r b 2^{--}\right)$mice and $B d k r b 2^{--}$mice treated with BK B1 receptor antagonist R715 in the same skin wound assay. There was no neutrophil recruitment defect in $\mathrm{Bdkrb2} 2^{-/-}$mice or $\mathrm{Bdkrb2^{-/- }}$ mice treated with B1R antagonist on either day $2(P=0.74$ among all 4 groups by 1-way ANOVA with Bonferroni's correction; Supplemental Figure $2 \mathrm{~A})$ or day 5 ( $P=0.18$; Supplemental Figure $2 \mathrm{~B})$.

Characterization of the role of FXII in sterile peritonitis. Thioglycolate-induced (TG-induced) peritonitis assay was used as a second model of sterile inflammation to examine leukocyte infiltration (peritoneal exudative cells [PEC]) into the peritoneum. Peritoneal lavage fluid from $F 12^{-/-}$mice contained significantly fewer PECs at 4 hours (Figure 2A). The peritoneal exudate differential count showed that $F 12^{-/-}$mice contained a significantly lower percentage of neutrophils compared with WT mice (Figure 2, B and C). On flow cytometry, the CD11b-positive, F4-80-negative cells, corresponding to the neutrophil population, also were significantly fewer in peritoneal exudate fluid from $\mathrm{F}^{-/-}$mice (9\%) compared with WT mice (39\%) (Figure 2D). In contrast, the number of PECs at 72 hours, representing macrophage recruitment, was not different among WT and $F 12^{-/-}$mice $(P=0.13$; Supplemental Figure 3$)$. These combined data indicate that FXII deficiency was associated with decreased neutrophil infiltration at sites of sterile inflammation. This finding was independent of reduced BK formation.

FXII deficiency results in improved wound repair. Studies next determined how the reduced inflammatory response seen in $F 12^{-/-}$mice translates in vivo in wound-healing potential. WT and $\mathrm{F} 12^{-/}$mice were wounded, and wound closure was monitored daily. Wounds in $\mathrm{F}^{-/-}$mice closed earlier than WT wounds (Figure $3 \mathrm{~A})$. When the wound area was measured daily from equally sized biopsies, $F 12^{-/}$mice had significantly smaller wounds compared with WT mice (Figure 3B). By day 7, 25\% of $F 12^{-/-}$wounds were completely closed (defined as wound area relative to day 0 of less than $5 \%$ ) compared with $0 \%$ of WT wounds (Figure 3B). By 8 days, wounds were completely closed in all $\mathrm{F} 12^{-/-}$mice compared with $25 \%$ of WT wounds $(P=0.02)$. To further evaluate wound healing, the reepithelialization of day 5 wounds was examined (Figure 3C). The mean percentage of reepithelialization in $\mathrm{F}^{-12^{--}}$wounds
$(70.3 \% \pm 7 \%$, mean $\pm \mathrm{SEM})$ was significantly greater than that in WT mice $(54.1 \% \pm 4 \%)(P=0.04)$ (Figure 3D). In early stages of wound healing, leukocyte recruitment, mainly of neutrophils, overlaps with keratinocyte proliferation $(29,30)$ and can interfere with healing. Immunohistochemistry of day 2 wounds showed decreased neutrophil elastase (NE) in the scab area and in the wound bed (Figure 3, E and F) and reduced citrullinated histone $\mathrm{H} 3$ (H3Cit), a NET marker, in $\mathrm{F} 12^{-/-}$wounds when compared with WT (Figure 3, G and H). Our data demonstrated that the reduced neutrophil recruitment seen in $F 12^{-/-}$mice had a beneficial effect on wound healing.

Targeting hepatic FXII does not influence neutrophil function. In order to determine whether FXII directly contributes to leukocyte function, FXII production in the liver was targeted by F12 siRNA. Initial kinetic experiments determined that $F 12$ siRNA treatment reduced plasma FXII coagulant activity to less than 5\% within 24 hours compared with control luciferase siRNA (Figure 4A). The half-life for plasma FXII decay, defined as the period of time required for FXII coagulant activity to be reduced by one-half (50\%), was 6.7 hours. Coincident with reduced FXII coagulant activity, plasma levels of FXII, as detected by immunoblot analysis, also became significantly reduced 24 hours after F12 siRNA treatment (Figure 4B; see complete unedited blots in the supplemental material). To assess the specificity of F12 siRNA, $0.5-\mathrm{cm}^{3}$ sections of liver were collected 24 hours after F12 and luciferase siRNA treatment, mRNA was isolated, and factors VII (FVII), IX (FIX), XI (FXI), and prekallikrein (PK) mRNA levels were quantified by real-time PCR (Figure 4C). F12-directed siRNA significantly reduced F12 mRNA expression ( $P=0.0013$; Figure $4 \mathrm{C}$ ). However, similarly to luciferase siRNA, F12 siRNA did not affect mRNA expression of FVII, FIX, FXI, and PK (Figure 4C). Therefore, the F12 siRNA specifically silenced F12 mRNA and protein levels as well as plasma clotting activity. Studies next determined whether siRNA treatment influenced leukocyte function. Unlike $F 12^{-/-}$mice, F12 siRNA-treated mice did not exhibit significant reduction in neutrophil recruitment in day $2(P=0.9$ vs. WT; Figure $4 \mathrm{D})$ or day 5 ( $P=0.1$ vs. WT; Figure $4 \mathrm{E})$ skin wounds. Similarly, in the TG peritonitis assay, $F 12$ siRNA-treated mice exhibited nor- 
A
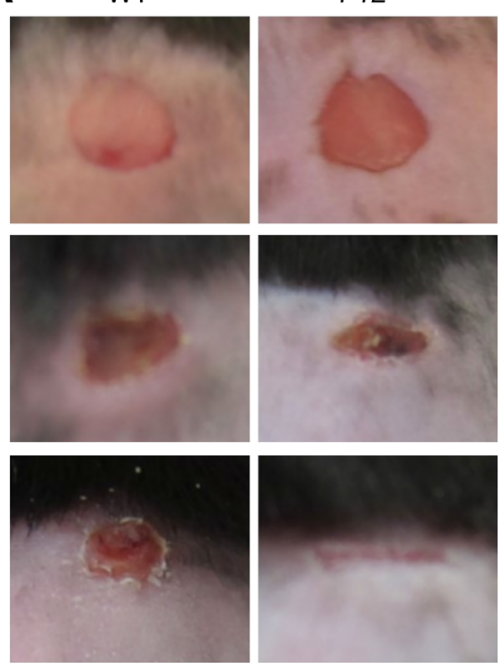

C

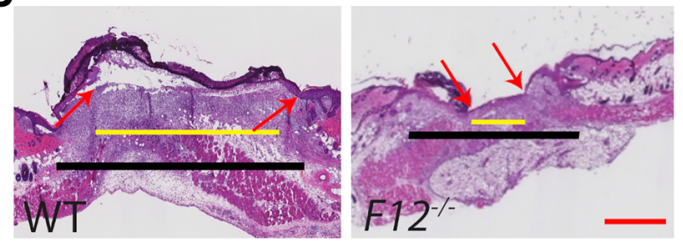

E

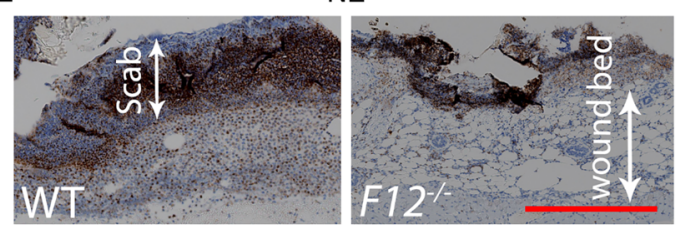

G

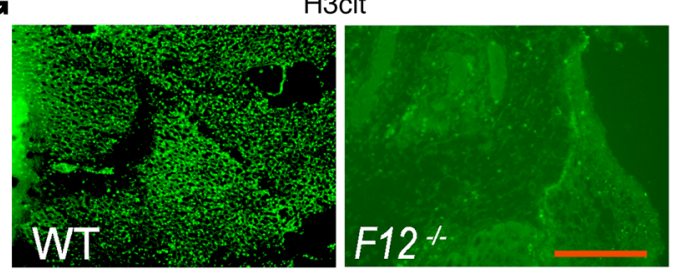

B

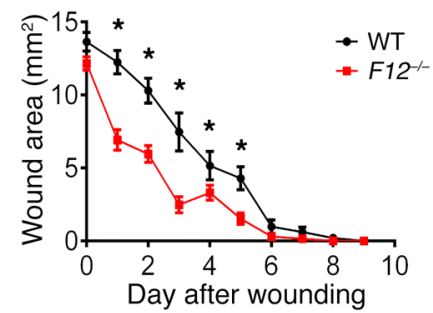

D

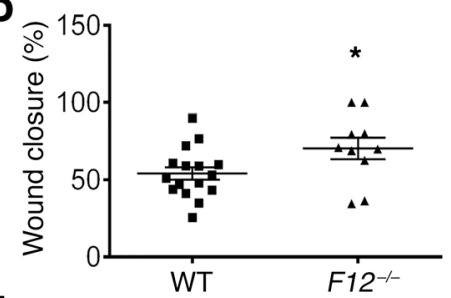

F

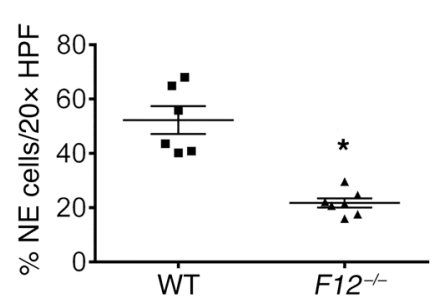

$\mathbf{H}$

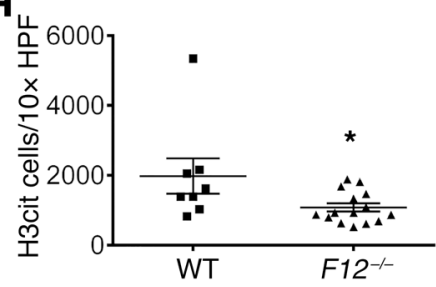

Figure 3. Influence of FXII on wound healing. (A) Full thickness wounds from WT and $F 12^{-1-}$ mice ( $n=12$ mice/group) were imaged daily until closure using a Nikon SMZ-U dissecting microscope. Original magnification, $\times 1$. (B) The wound area $\left(\mathrm{mm}^{2}\right)$ was measured daily from day 0 until closure in WT $(n=20)$ and $F 12^{-/-}$ $(n=28)$ mice. Wounds were considered closed when their areas relative to day 0 were less than $5 \%$. Mean \pm SEM. ${ }^{*} P<0.05$, 2-way ANOVA. (C) H\&E-stained sections from day 5 were analyzed to determine the degree of reepithelialization. Black line demarcates the total length of wound space; yellow line represents the remaining wound gap. Red arrows indicate the epithelial tongues showing ingrowing epithelium. All histologic sections were obtained using a Leica SCN 400 slide scanner equipped with a Hamamatsu line sensor color camera at $\times 4$ magnification. Scale bar: $100 \mu \mathrm{m}$. (D) The percentage of reepithelialization (\% wound closure) was determined using the Imagej software and is shown for WT $(n=16)$ and $F 12^{-/-}(n=10)$ mice. Mean \pm SEM. ${ }^{*} P<0.05$, Student's $t$ test. (E and $\mathbf{G}$ ) Sections from day 2 wounds were stained for $\mathrm{NE}(\mathbf{E})$ at $\times 20$ magnification or $\mathrm{H} 3 \mathrm{Cit}(\mathbf{G})$ at $\times 10$ magnification, respectively. Scale bars: $50 \mu \mathrm{m}$ (E); $20 \mu \mathrm{m}$ (G). (F) NE content was determined in the wound bed immediately beneath the scab in day 2 wounds and presented as percentage of NE-stained cells to total number of cells per high-power field. WT, $n=6 ; F 12^{-1-}$ $n=7$. Mean \pm SEM. ${ }^{*} P=0.001$, Student's $t$ test. (H) H3Cit content of day 2 wounds is presented as total number of cells per high-power field. WT, $n=8 ; F 12^{-1-}, n=15$. Mean \pm SEM. ${ }^{*} P<0.04$, Student's $t$ test. mal PEC counts, as did WT mice treated with control luciferase siRNA (Figure 4F). Infusion of human FXII into $\mathrm{F} 12^{-/}$mice such that the plasma FXII concentration was made physiologic at 450 $\mathrm{nM}$ did not correct the PEC migration defect seen in $\mathrm{F} 12^{-/}$mice (Figure $4 \mathrm{G}$ ). We also reconstituted $F 12^{--}$mice with $675 \mathrm{nM}$ of recombinant murine FXII (mFXII), corresponding to 1.5 times the plasma FXII concentration. We immediately challenged these animals with peritoneal TG instillation and performed peritoneal lavage and plasma collection 4 hours later. Based on our kinetic experiments, which show the mFXII half-life to be 6.7 hours, we rationalized that the reconstituted FXII concentration at the time of harvest ( $t=4$ hours) would be sufficiently high to support any role of circulating FXII in neutrophil infiltration. FXII coagulant activity in mice reconstituted with $\mathrm{mFXII}$ at the time of peritoneal lavage ( $t=4$ hours) was $70.2 \% \pm 4.4 \%$, and prothrombin time was normal (Supplemental Figure 4, A and B). However, as shown in
Figure 4G, normal levels of mFXII, like human FXII, were unable to correct the neutrophil infiltration defect in $\mathrm{F}^{2 /-}$ mice. These studies indicate that the neutrophil migration defect in $\mathrm{F}_{12-/}$ mice is not dependent on plasma FXII.

FXII cDNA and protein in murine and human neutrophils. Investigations next determined whether FXII was expressed in neutrophils. Murine total mRNA was isolated from BM-derived neutrophils and homogenized livers, and first-strand cDNA was synthesized. A representative PCR product of approximately $345 \mathrm{bp}$ is shown in Figure 5A; see complete unedited blots in the supplemental material. There was complete sequence homology between murine neutrophil- and hepatic-derived $F 12$ in all 14 exons queried. Neutrophil F12 mRNA expression is approximately 4 -fold lower than hepatic F12 expression (Figure 5A) (31). PCR analysis of F12 siRNA-treated neutrophils showed preserved F12 expression in these cells (Supplemental Figure 
A

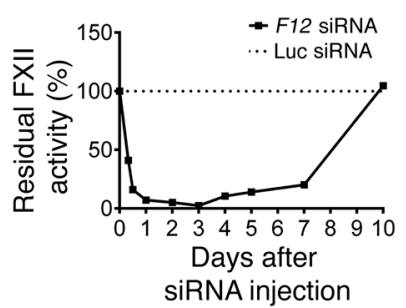

E

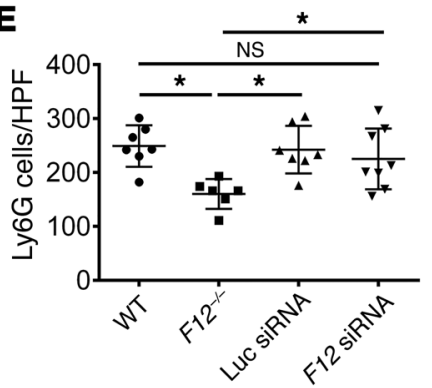

B

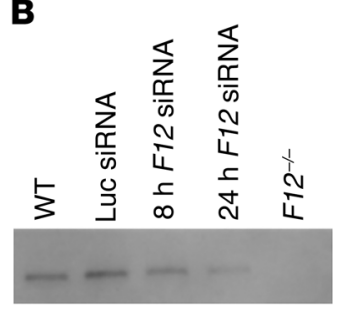

$\mathbf{F}$

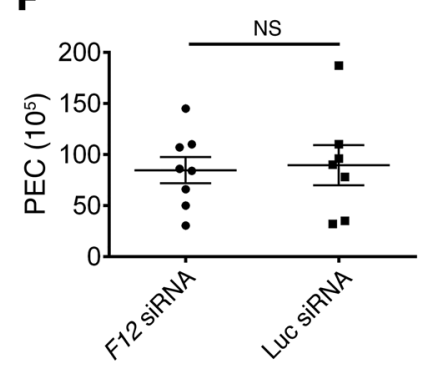

C

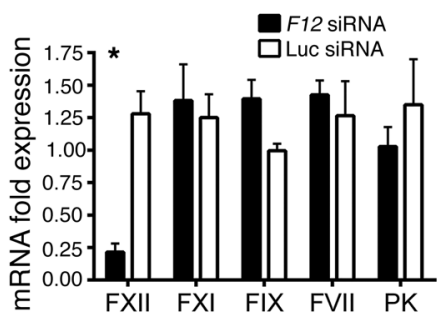

G

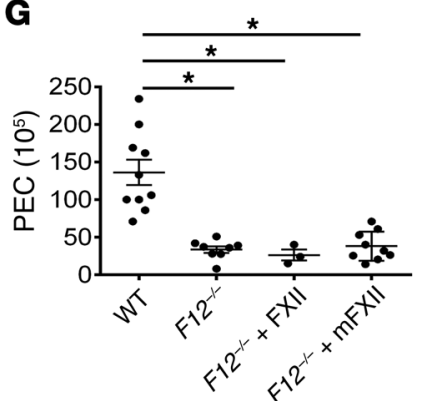

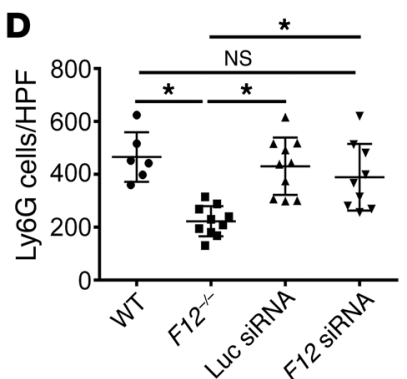

Figure 4. Influence of F12 siRNA on inflammation and thrombosis. (A) Plasma FXII coagulant activity following siRNA treatment. WT mice $(n=6 /$ time point) were treated by tail-vein injection with $F 12$ or luciferase siRNA at $0.1 \mathrm{mg} / \mathrm{kg}$ dose. At indicated times, blood was collected and residual plasma FXII activity was determined by an aPTT-based assay. (B) Western blot for FXII was performed under reduced conditions 8 hours and 24 hours after F12 siRNA treatment. Representative blot of $n=4$. (C) WT mice were treated intravenously with luciferase (Luc siRNA) or F12 siRNA. Twenty-four hours after dosing, liver was collected for hepatic mRNA expression. Note the specificity of $F 12$ siRNA for $F 12$ gene silencing. Mean $\pm S E M$. $n=5$ individual experiments run in triplicate. ${ }^{*} P=0.001$ by mean row statistics. ( $\mathbf{D}$ and $\mathbf{E}$ ) Twenty-four hours after siRNA treatment, mice received 5 -mm full-thickness punch biopsies. Wounds were harvested on day 2 and day 5, and frozen sections were stained with anti-Ly6G antibody. (D) Number of Ly6G cells/HPD in day 2 wounds. WT, $n=6$; F12 ${ }^{-1-}, n=10$; Luc siRNA treated, $n=10 ; F 12$ siRNA treated, $n=9$. Mean \pm SEM. ${ }^{*} P<0.006,1$-way ANOVA. (E) Number of Ly6G cells/ high-power field in day 5 skin wounds. WT, $n=7 ; F 12^{-1-}, n=6$; Luc siRNA treated, $n=7$; F12 siRNA treated, $n=8$. Mean \pm SEM. ${ }^{*} P<0.02,1$-way ANOVA. (F) WT mice were treated with F12 siRNA $(n=8)$ or luciferase siRNA $(n=7)$, and 24 hours later, they underwent TG-induced peritonitis. PEC number was determined at 4 hours. Mean \pm SEM. $P=0.837$ by Student's $t$ test. (C) PEC number 4 hours after TC instillation in WT $(n=10), F 12^{-/-}(n=8)$, and $F 12^{-/-}$ mice reconstituted with purified human FXII $(n=3)$ or recombinant mouse FXII (mFXII) $(n=9)$ to physiologic plasma FXII levels of $450-650 \mathrm{nM}$. Mean \pm SEM. ${ }^{*} P \leq 0.0003$ vs. WT, 1-way ANOVA.

$5 \mathrm{~A}$; see complete unedited blots in the supplemental material). Peripheral blood murine neutrophils contained FXII on immunofluorescence (Figure 5B). Confocal microscopy studies with normal human neutrophils determined that in resting (untreated [UT]) cells, FXII was distributed throughout the cell (Figure $5 C)$. When neutrophils were activated with $\mathrm{N}$-formylmethionyl peptide (fMLP), FXII translocated to the plasma membrane on midoptical confocal microscopy projections (Figure 5C). No FXII was seen in peripheral neutrophils from a patient with congenital FXII deficiency (Hageman trait) (Figure 5D) or in neutrophils from $\mathrm{F}^{2-} \%$ mice (Figure $5 \mathrm{~B}$ ). Immunoblot analysis of resting or fMLP-stimulated monocytes did not show the presence of FXII protein (Supplemental Figure 5B; see complete unedited blots in the supplemental material).

FXII signals through uPAR to stimulate $p A k t S^{473}$ and $p A k t 2 S^{474}$ in neutrophils. Studies next examined how neutrophil FXII influences cell activities. First, when washed neutrophils were stimulated with fMLP for 1 to 5 minutes, cells and supernatant were separated and resolved on SDS-PAGE and FXII was detected with FXII-specific antibody. FXII was present in UT cells, but not in supernatant from these cells (Figure 6A; see complete unedited blots in the supplemental material). Upon fMLP stimulation, FXII content decreased in the cell fraction and increased in the supernatant over time (Figure $6 \mathrm{~A})$. These data showed that following neutrophil activation,
FXII was secreted. Since UPAR is functionally important for leukocyte activities (32-36) and serves as a receptor for FXII on endothelial cells (22), we performed surface plasmon resonance studies, which showed that FXII bound to uPAR in a concentration-dependent manner (Figure 6B). Binding kinetics showed a high-affinity interaction with $k_{\text {on }}$ (association rate constant) of $2.03 \pm 0.85 \times 10^{5}$ $\mathrm{M}^{-1} \mathrm{~s}^{-1}, k_{\text {off }}$ (dissociation rate constant) of $5.02 \pm 2.82 \times 10^{-3} \mathrm{~s}^{-1}$, and $K_{D}$ (equilibrium dissociation constant) of $37.1 \pm 29.4 \mathrm{nM}$ (Supplemental Table 2). BiaCore studies also showed that the FXII-uPAR interaction requires $\mathrm{Zn}^{2+}$ ions (Figure 6B). Collectively, these findings confirmed that FXII and UPAR interact on the neutrophil surface in a zinc-dependent manner.

Investigations next determined whether the interaction between FXII and UPAR results in neutrophil activation. Since several neutrophil functions depend on pAktS ${ }^{473}$ signaling (37, 38), we observed that fMLP or FXII/ $/ \mathrm{Zn}^{2+}$ treatment of neutrophils increased AktS ${ }^{473}$ phosphorylation in WT neutrophils (Figure 6C; see complete unedited blots in the supplemental material). FXII-induced pAktS ${ }^{473}$ was blocked upstream at PI3K by Wortmannin and LY294002 (Figure 6C). Similar results were obtained with murine $\mathrm{F} 12^{--}$neutrophils (Figure 6D; see complete unedited blots in the supplemental material). Although fMLP-induced pAktS ${ }^{473}$ in uPAR-deficient (Plaur ${ }^{-}$) neutrophils was partially reserved, no FXII/ $\mathrm{Zn}^{2+}$-induced pAktS ${ }^{473}$ was observed in Plaur ${ }^{-1-}$ 

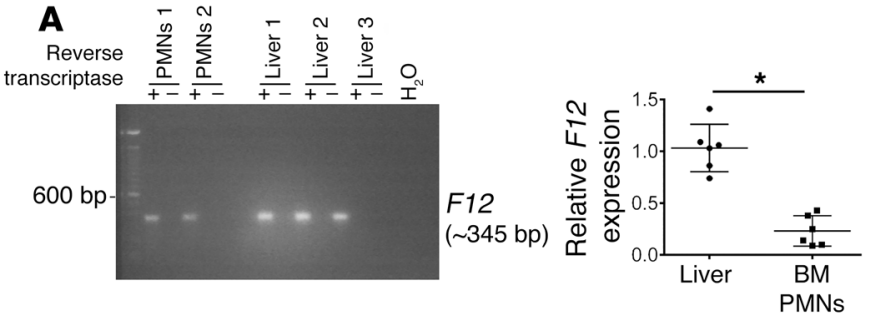

B
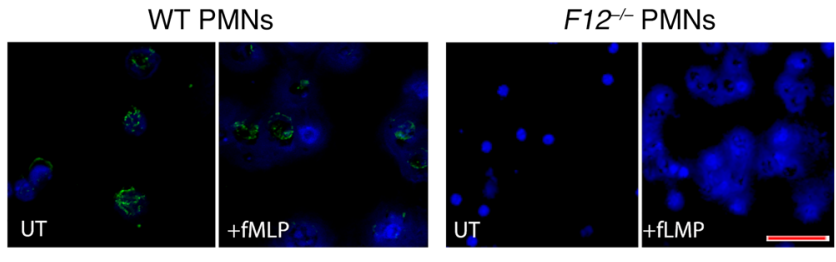

\section{C}

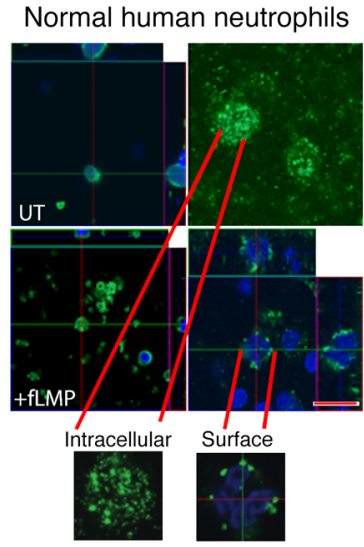

D

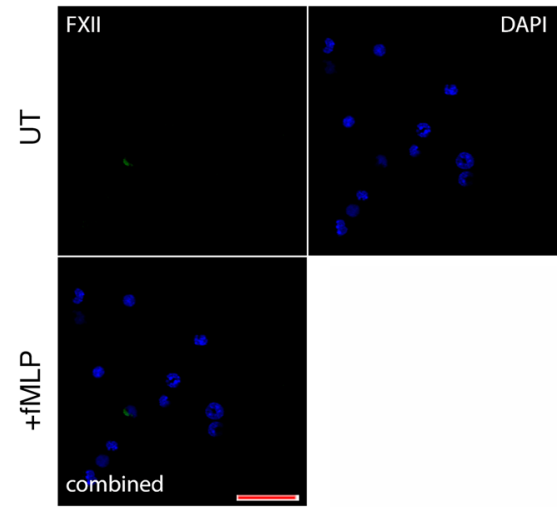

Figure 5. Neutrophils (PMNs) express F12 mRNA and FXII. (A) Left panel: murine total mRNA was isolated from BM-derived neutrophils (PMNs 1,2) or homogenized livers (Liver 1-3), and first-strand cDNA was synthesized with SuperScript III reverse transcriptase. The PCR product from the same animal on an exon 1-6 probe is shown. Images are representative of 3 experiments. Right panel: relative $F 12$ expression in liver and BM-derived neutrophils as determined by $2^{-\Delta \Delta c t}$. Mean \pm SEM. ${ }^{*} P=0.0001$, Student's $t$ test. (B) Isolated WT (left panel) and $F 12^{-/-}$(right panel) murine peripheral blood neutrophils were incubated with media or fMLP for 2 hours. Cells were stained with primary antibody directed against FXII (green); nuclei were counterstained with DAPI (blue). Images are representative of $n=3$ experiments. Original magnification, $\times 20$. Scale bar: $10 \mu \mathrm{m}$. (C) Confocal visualization of FXII in normal human peripheral neutrophils. Neutrophils were incubated with media (UT; top panels) or fMLP (bottom panels), then either not permeabilized (surface) or permeabilized (intracellular) and stained with antibody to FXII. Confocal microscopy indicates the intracellular location of FXII protein before treatment and trafficking to the cell surface after stimulation with $\mathrm{fMLP}$. Images shown are representative of 3 individual experiments. Original magnification, $\times 20$ (left panel); $\times 100$ (right panel). Scale bar: $5 \mu \mathrm{m}$. (D) Confocal FXII visualization in neutrophils from FXII-deficient patient in UT (top) and fMLP-treated (bottom) cells. FXII is absent in peripheral neutrophils from a patient with congenital FXII deficiency. Original magnification, $\times 10$. Scale bar: $10 \mu \mathrm{m}$.

neutrophils (Figure 6E; see complete unedited blots in the supplemental material). In human and mouse neutrophils, Akt2 is the predominant Akt isoform that regulates neutrophil functions $(37,39,40)$. We observed that in WT neutrophils, pAkt2S ${ }^{474}$ was substantially increased in response to fMLP and FXII/ $/ \mathrm{Zn}^{2+}$ (Figure $6 \mathrm{~F}$; see complete unedited blots in the supplemental material). In F12 $2^{--}$neutrophils, both fMLP and FXII/Zn ${ }^{2+}$ promoted pAkt2S ${ }^{474}$ as well (Figure 6G; see complete unedited blots in the supplemental material). However, FXII/ $\mathrm{Zn}^{2+}$ did not promote Akt2S ${ }^{474}$ phosphorylation in Plaur ${ }^{-}$neutrophils (Figure 6H; see complete unedited blots in the supplemental material). When we treated neutrophils with a specific Akt2 inhibitor (Akti XII) that is highly selective for Akt2 over Akt1/Akt3 (41), FXII-mediated pAkt2S ${ }^{474}$ of murine WT and $F 12^{--}$neutrophils was significantly inhibited by Akti XII (Figure 6, F and G; see complete unedited blots in the supplemental material). When the intracellular $\mathrm{Zn}^{2+}$ ions were sequestered by pretreating cells with the membrane-permeable $\mathrm{Zn}^{2+}$-selective chelator TPEN, reduced Akt2 $\mathrm{S}^{474}$ phosphorylation was also observed with FXII/Zn ${ }^{2+}$ (Figure 6, F and G; see complete unedited blots in the supplemental material). These latter data indicate that, in neutrophils, FXII-induced Akt2 phosphorylation requires intracellular $\mathrm{Zn}^{2+}$.
FXII-mediated signaling in neutrophils is a zymogen function. We next determined whether the effect of FXII on neutrophils is a zymogen property or an enzymatic activity. WT neutrophils were stimulated with FXII and $\mathrm{Zn}^{2+}$ for up to 5 minutes. We determined that exogenous FXII remained a single chain $(\sim 78 \mathrm{kDa})$ on reduced SDS-PAGE (Figure 7A; see complete unedited blots in the supplemental material). In a chromogenic assay measuring S-2302 cleavage, WT neutrophils were incubated in the absence or presence of fMLP, FXII/ $\mathrm{Zn}^{2+}$, or increasing concentrations of FXIIa $(0.62 \mathrm{nM}, 6.2 \mathrm{nM}, 62.5 \mathrm{nM})$ and $\mathrm{Zn}^{2+}$. We found no significant amidolytic activity in UT or fMLP-treated neutrophils (Figure 7B). S-2302 cleavage in FXII/ $\mathrm{Zn}^{2+}$-treated cells progressed at a slow rate and was not significant at 5 minutes, but reached statistical significance at later time points (Figure 7C). In contrast, there was a concentration-dependent increase in S-2302 hydrolysis when FXI$\mathrm{Ia} / \mathrm{Zn}^{2+}$ was incubated with neutrophils (Figure $7 \mathrm{~B}$ ). This increased amidolytic activity started as early as 5 minutes ( $62.5 \mathrm{nM}$ of FXIIa) and persisted at 180 minutes ( $6.2 \mathrm{nM}$ and $62.5 \mathrm{nM}$ of FXIIa) of cell incubation with FXIIa/ $\mathrm{Zn}^{2+}$ (Figure 7C). These data show that FXII does not exhibit early enzymatic activity on the surface of neutrophils, especially not within the 5 minutes that is the end point in pAkt2 signaling studies. 
A

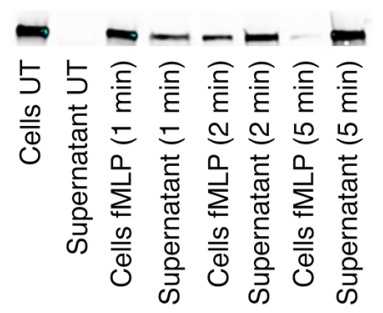

C

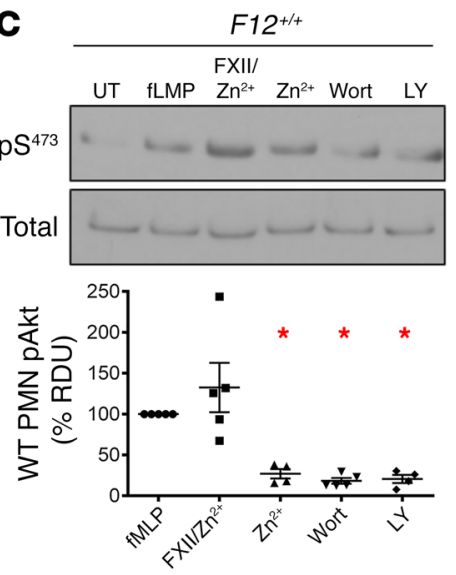

$\mathbf{F}$
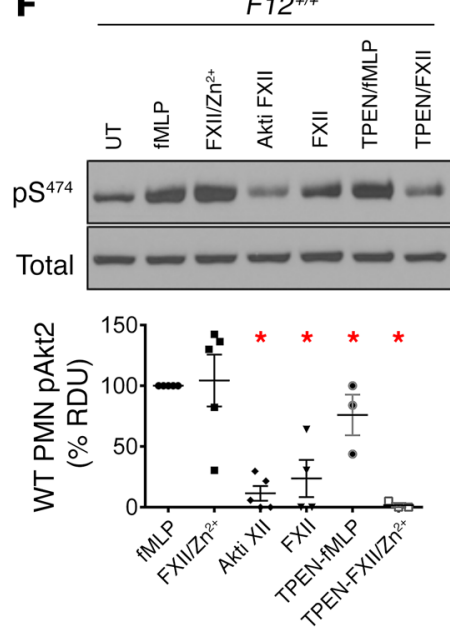

B

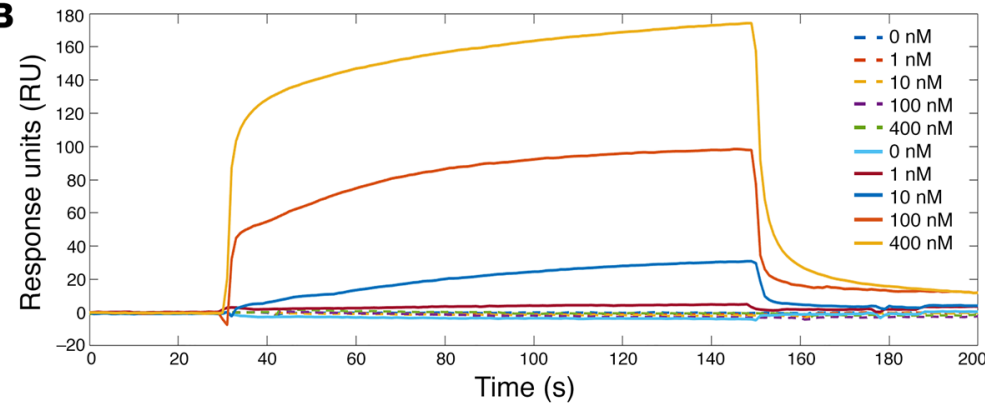

D

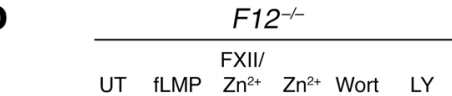

$\mathbf{E}$
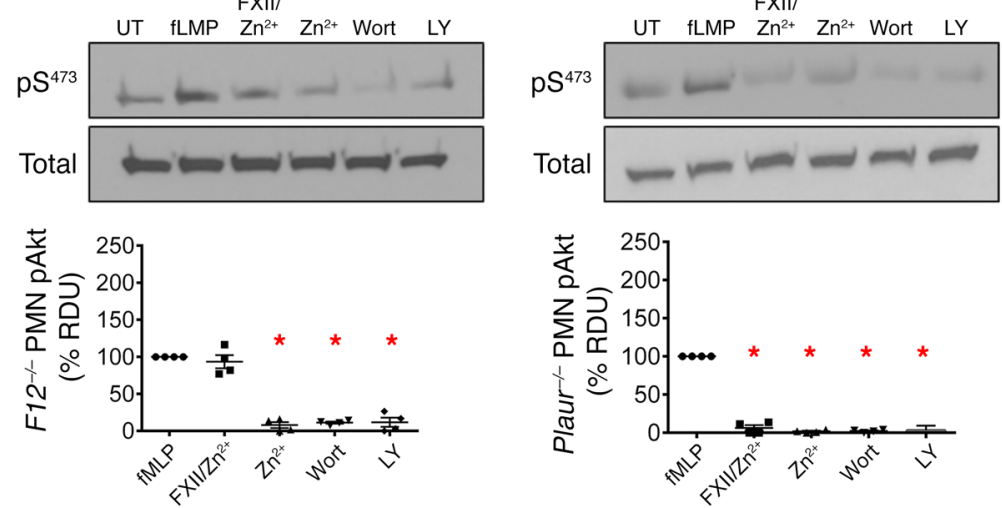

G

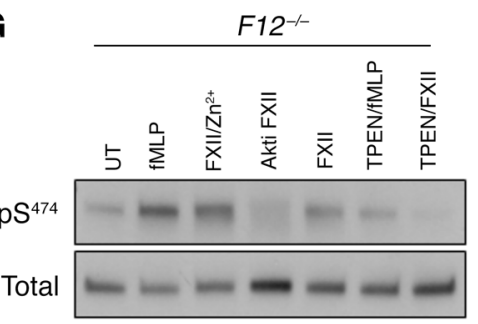

H
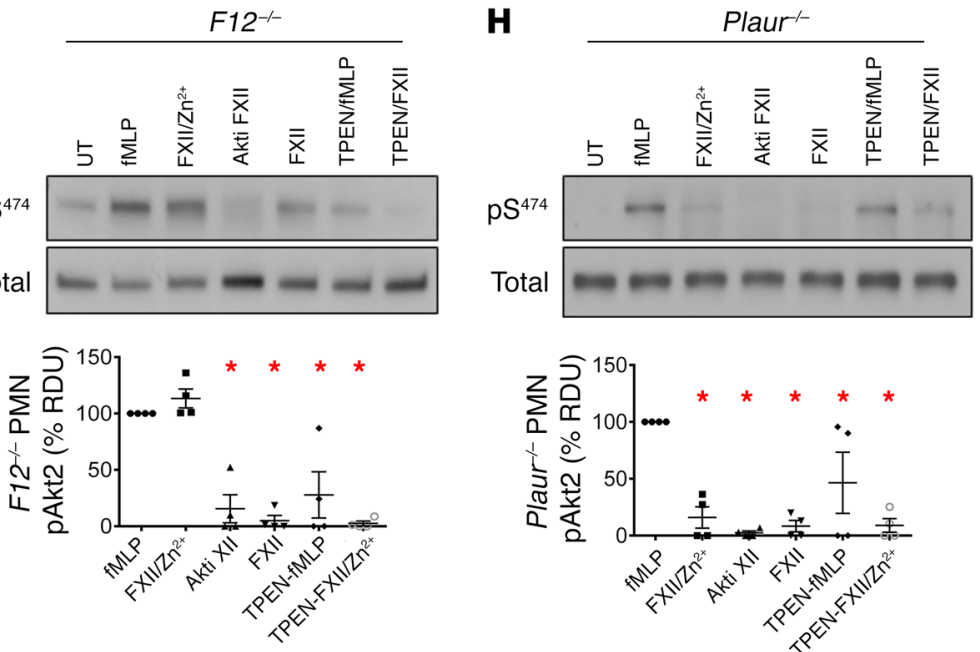

Figure 6. FXII is secreted from neutrophils and signals through uPAR to promote pAkt5 ${ }^{473}$ and pAktS ${ }^{474}$. (A) WT neutrophils (PMNs) were incubated in the absence or presence of fMLP. At indicated time points, cells and supernatant were separated and resolved on SDS-PACE. FXII was detected with antiFXII antibody. Representative blot of $n=3$. (B) Sensorgram of FXII binding to immobilized uPAR in the presence (solid lines) or absence (dotted lines) of $10 \mu \mathrm{ZZn}^{2+}$. Increasing concentrations of FXII $(0 \mathrm{nM}, 1 \mathrm{nM}, 10 \mathrm{nM}, 100 \mathrm{nM}, 400 \mathrm{nM})$ were injected over a uPAR-immobilized, gelatin-coated CM5 chip. Each sensorgram line represents an average of 3 injection runs as described in Methods. Analyte injection was terminated at 120 seconds. (C-E, top panels) PMNs were treated with $\mathrm{fMLP}, \mathrm{FXII} / \mathrm{Zn}^{2+}$, or $\mathrm{Zn}^{2+}$ alone for 5 minutes, followed by immunoblotting. Wort or LY lanes were pretreated with Wortmannin (50 $\mu \mathrm{M})$ and LY294002 (100 $\mu \mathrm{M})$ for 1 hour, respectively, followed by FXII/Zn ${ }^{2+}$ treatment. (C-E, bottom panels) Percentage pAktS ${ }^{473}$ in neutrophils. (F-H, top panels) Neutrophils were treated with fMLP, FXII/Zn ${ }^{2+}$, or FXII alone. Lanes labeled Akti were pretreated with Akti XII (5 $\left.\mu \mathrm{M}\right)$ for 30 minutes, followed by $\mathrm{FXII} / \mathrm{Zn}^{2+}$ treatment. Where indicated, cells were pretreated with TPEN $(10 \mu \mathrm{M})$ for 30 minutes before stimulation with $\mathrm{fMLP}$ or FXII/Zn ${ }^{2+}$. Lysates were immunoblotted with antibodies against pAkt2S $S^{474}$. (F-H, bottom panels) Percentage of pAkt2S $S^{474}$ (\% relative density units [RDU]) in neutrophils. UT cell band density was considered $0 \%$; band density of fMLP-treated cells subtracted from UT band density was set at $100 \%$. Data in panels D-I represent mean \pm SEM of 4 or more experiments. ${ }^{*} P<0.0001,1$-way ANOVA.

Since recent investigations show that single-chain FXII exhibits weak proteolytic activity, we generated a double FXII mutant, termed FXII-D, that consists of 2 mutations: FXII locarno (FXIIR353P mutation) (8) and FXII-S544A (an alanine substituting the active site serine). On reduced SDS-PAGE, FXII WT, FXII-D, purified plasma-derived XII (purified FXII), and FXII locarno migrated similarly on gel electrophoresis (Figure 7D; see complete unedited blots in the supplemental material). FXII-D lacks 

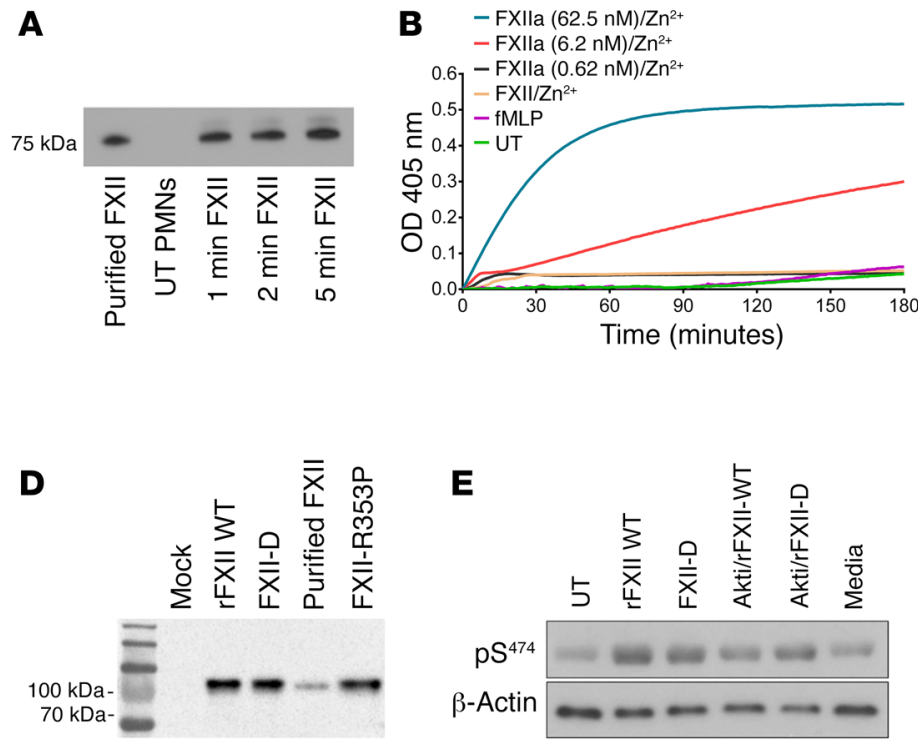

C
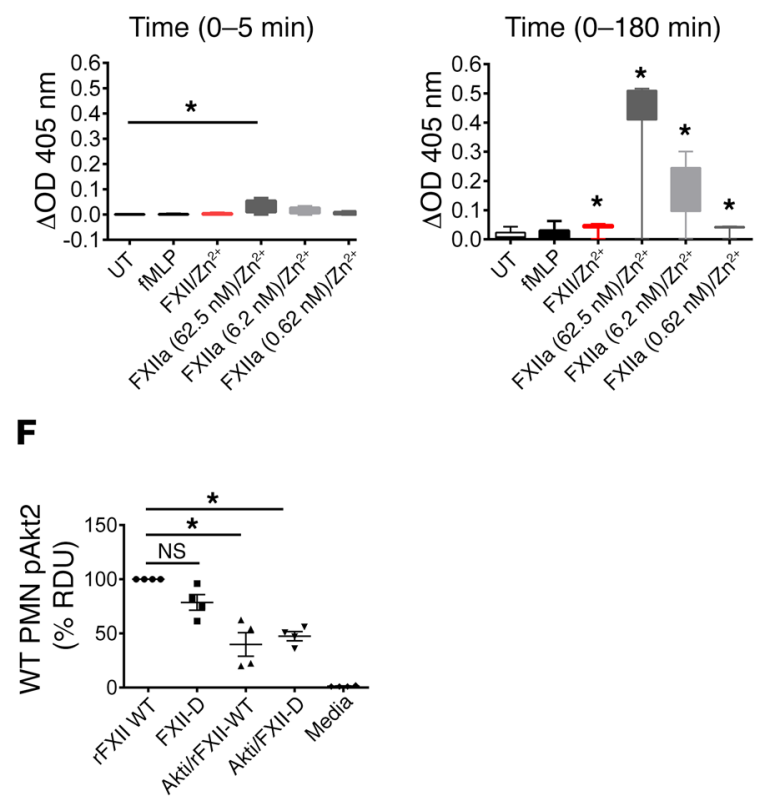

Figure 7. FXII signaling in neutrophils is a zymogen function. (A) WT neutrophils were incubated in the absence (UT) or presence of $62 \mathrm{nM}$ FXII and $10 \mu \mathrm{M}$ $\mathrm{Zn}^{2+}$ for 1, 2, and 5 minutes. Western blot analysis for FXII was performed under reduced conditions using polyclonal anti-FXII antibody. Representative blot of $n=3$. (B) Chromogenic assay of FXIla activity. Neutrophils were allowed to adhere on a gelatin-coated 96 -well plate for 10 minutes and subsequent-

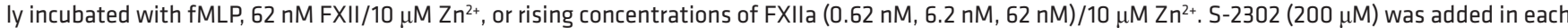
well, and changes in $\mathrm{OD}_{405}$ were continuously monitored on a microplate reader. Graph represents mean $\mathrm{OD}$ of a single experiment run in triplicate. (C) Left panel: cleavage of S-2302 is presented as change in mean OD $(\triangle \mathrm{OD})$ over the first 5 minutes of neutrophil incubation with media, $\mathrm{fMLP}, \mathrm{FXII} / \mathrm{Zn}{ }^{2+}$, or rising concentrations of FXIla/ $\mathrm{Zn}^{2+}$. Note that 5 minutes is the time point used in pAkt2S $\mathrm{S}^{474}$ signaling studies. Right panel: cleavage of S-2302 over 180 minutes. Graphs are presented in box-and-whiskers diagrams where minimum/maximum distribution and outliers are shown. Mean $\triangle O D, n=4$ individual experiments run in triplicate. (D) FXII immunoblotting of supernatant from HEK293 cells expressing WT FXII, FXII Locarno (FXII-R353P), and FXII double-mutant (FXII-D) (combined R353P and S544 substitutions). Mock: supernatant from nontransfected cells; Purified FXII: plasma-derived mouse FXII. (E) Neutrophils were treated with WT FXII/ Zn ${ }^{2+}$ or FXII-D variant and Zn $n^{2+}$. Lanes labeled Akti were pretreated with Akti XII (5 $\mu$ M) for 30 minutes, followed by FXII/ $\mathrm{Zn}^{2+}$ or FXII-D/Zn ${ }^{2+}$. Lysates were immunoblotted with antibodies against pAkt25 ${ }^{474}$. Media: conditioned media from transfected cells. (F) Percentage of pAkt2S $S^{474}$ (\% RDU) in neutrophils. Mean \pm SEM, $n=4$ experiments. ${ }^{*} P<0.0001,1$-way ANOVA.

all enzymatic activity and is incapable of contact-activation. $F 12^{-/-}$ plasma reconstituted with WT FXII shortened the aPTT to normal range $(35.8 \pm 1.2$ seconds, mean \pm SEM; Supplemental Figure 6). In contrast, when FXII-D was added to $F 12^{-/-}$plasma, the aPTT remained significantly prolonged ( $>220$ seconds, which is the upper limit of recording times; Supplemental Figure 6). However, independently of its inability to generate coagulant or enzymatic activity, FXII-D was able to promote Akt2 phosphorylation in neutrophils (Figure 7, E and F; see complete unedited blots in the supplemental material). These studies, along with the kinetic enzymatic studies above, show that FXII-mediated signaling in neutrophils is a zymogen FXII function.

The FXII/uPAR axis contributes to key cell functions. Since FXII signals in neutrophils and $F 12^{-1-}$ mice had reduced inflammatory responses, we determined whether FXII regulates cell activities. First, neutrophil adhesion to fibrinogen was examined in WT and $F 12^{-/}$cells. After fMLP stimulation, $F 12^{-/-}$neutrophil adhesion to fibrinogen was significantly reduced, suggesting that neutrophil FXII itself is important for adhesion (Figure 8A). Chemotaxis was examined with Boyden chambers using fMLP or FXII as a gradient. $F 12^{-/}$neutrophil migration was significantly lower than that of WT cells regardless of gradient (Figure 8B). Additionally, FXII itself in the presence of $\mathrm{Zn}^{2+}$ was a chemotaxin to normal neutro- phils, but this function was reduced with $\mathrm{F} 12^{-/-}$neutrophils (Figure $8 \mathrm{~B})$. To further determine whether FXII promoted directional cell migration, a kinetic chemotaxis assay was developed, employing a microfluidic chamber and time-lapse microscopy (Supplemental Figure 7) (42). In these studies, $F 12^{-/-}$neutrophils overall had less chemotaxis than WT cells for both chemoattractants, fMLP and FXII/Zn ${ }^{2+}$ (Supplemental Figure 8 and Supplemental Video 1). By examining the rate of chemotaxis, FXII was a more potent chemoattractant for WT cells than fMLP (Figure 8, C and D). However, for both chemotaxins, $F 12^{-/-}$neutrophils had about half the chemotaxis rate of WT neutrophils (Figure 8, E and F). Finally, uPAR appeared to mediate FXII-induced chemotaxis, since Plaur/- neutrophils had no significant chemotaxis in response to FXII/ $\mathrm{Zn}^{2+}$ and exhibited significantly less migration in response to fMLP (approximately 25\% that of WT neutrophils) (Figure 8, G and H).

FXII/UPAR upregulates $\alpha_{M} \beta_{2}$ integrin, increases intracellular $\mathrm{Ca}^{2+}$ mobilization, and promotes NET formation. We further dissected how the FXII/uPAR axis contributes to neutrophil activities. We describe above that $F 12^{-/-}$cells adhere significantly less onto fibrinogen (Figure 8A). Neutrophil adhesion to extracellular matrices and cells is an integrin-dependent function $(43,44)$. It was previously shown that the surface expression of $\alpha_{M} \beta_{2}$, but not $\alpha_{\mathrm{L}} \beta_{2}$, integrin is significantly reduced in Akt2-KO neutrophils upon 

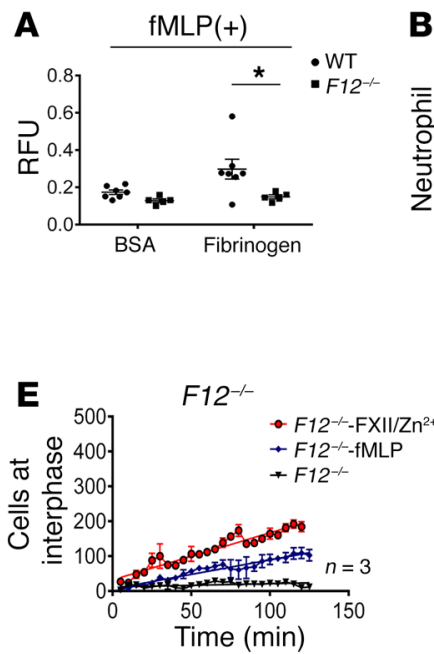

B

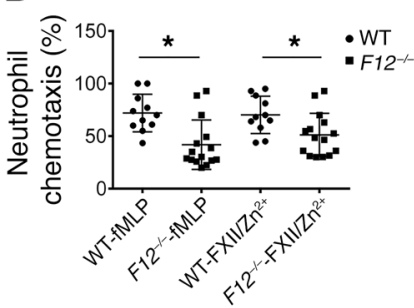

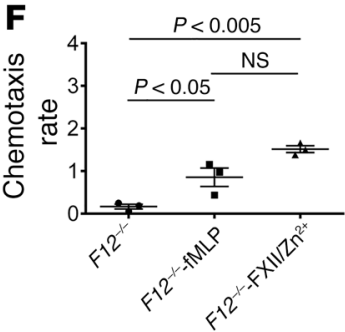

C
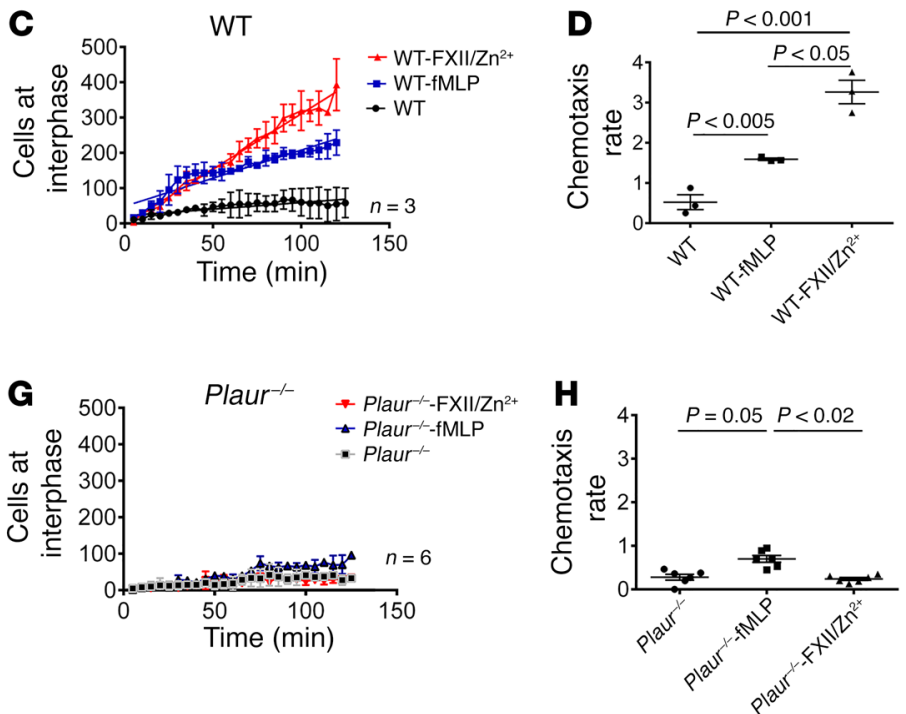

Figure 8. The FXII-uPAR axis promotes neutrophil adhesion and chemotaxis. (A) WT $(n=7)$ and $\mathrm{F} 12^{-/-}(n=5)$ peripheral neutrophils $\left(1 \times 10^{6} / \mathrm{ml}\right)$ were incubated with fMLP and applied on BSA- or fibrinogen-coated 96 -well plates. Adhered cells were determined by a fluorogenic assay reported in relative fluorescence units (RFU), $480 / 520 \mathrm{~nm}$. Mean \pm SEM. ${ }^{*} P<0.02 \mathrm{vs}$. WT neutrophils by Student's $t$ test. (B) Boyden chamber chemotaxis. Medium alone,

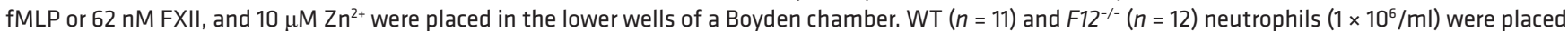
in the upper wells. Chemotaxis was determined by measuring fluorescence in the lower wells. Mean $\pm \mathrm{SEM}$ vs. $\mathrm{fMLP}$ and FXII/Zn ${ }^{2+}$ treatment(s). ${ }^{*} P<$ 0.0001 , 1-way ANOVA with Bonferroni's correction. (C-H) Microfluidic channel assay of live-cell chemotaxis. Murine neutrophils (1 $\left.\times 10^{6} / \mathrm{ml}\right)$ were applied onto Matrigel-coated microfluidic channels that contained an interphase with a pocket infused with $62 \mathrm{nM}$ FXII and $10 \mu \mathrm{M} Z \mathrm{n}^{2+}$, $10 \mu \mathrm{M} \mathrm{fMLP}$, or media. Images at the interphase were obtained every 5 minutes for 2 hours. WT (C), F12 ${ }^{-/-}$(E), and Plaur ${ }^{-/-}$(C). Cumulative linear regression curves of relative cell increase of neutrophils at the interphase over 2 hours. WT (D), F12 ${ }^{-/-}$(F), and Plaur ${ }^{-/-}$(H). Chemotaxis rates of neutrophils from each indicated genotype as determined by the slope of linear regression curves. Graphs plotted as mean \pm SEM, $n=3-6$ experiments. $P$ values in each panel were determined by $1-$ way ANOVA with Bonferroni's correction.

fMLP stimulation (39). Therefore, we asked whether FXII-uPARpAkt2 interactions specifically upregulate surface $\alpha_{M} \beta_{2}$ integrin. WT neutrophils were stimulated with fMLP or FXII $/ \mathrm{Zn}^{2+}$, and surface expression of $\alpha_{M} \beta_{2}$ was determined by flow cytometry. We found that, following FXII $/ \mathrm{Zn}^{2+}$ stimulation, the surface expression of $\alpha_{\mathrm{M}} \beta_{2}$ integrin was significantly increased ( $P=0.03 \mathrm{vs.} \mathrm{UT} \mathrm{neutro-}$ phils; Figure 9, A and B). We next asked whether FXII stimulation of neutrophils increases intracellular calcium. We focused on this specific question due to prior studies that showed that following neutrophil activation, Akt 2 is the only isoform that translocates to the leading edge of neutrophils, where it associates with the cytoskeleton (37). We employed a Fluo-4-based assay that measures cytosolic calcium concentration $\left(\left[\mathrm{Ca}^{2+}\right]\right)$. These data showed that stimulation of neutrophils with FXII/ $\mathrm{Zn}^{2+}$, similar to stimulation with fMLP, induced an approximately 2.3 -fold increase in cytosolic $\left[\mathrm{Ca}^{2+}\right]$ from the basal level of $27 \mathrm{nM}$; the change in $[\mathrm{Ca} 2+]$ peaked within 2 minutes and then modestly decreased during the next 30 minutes (Figure 9, C and D).

Immunofluorescence studies showed that $\mathrm{F} 12^{-/-}$wounds contained fewer NETs (Figure 3, G and H). This finding may simply be due to the reduced presence of neutrophils in skin wounds. However, NET formation is governed by 2 major signaling pathways, NOX2-dependent $(45,46)$ and NOX2-independent (mediated by mitochondrial ROS) (47) pathways, and both pathways require pAkt activity (47). Since FXII promotes Akt2 phosphorylation at $\mathrm{S}^{474}$, we asked whether FXII-mediated signaling in neutrophils itself promoted NETosis. Isolated WT neutrophils were stimulated with PMA, fMLP, and FXII/ $\mathrm{Zn}^{2+}$ for 2 hours. Immunoblot- ting was performed for H3Cit, a marker of NETosis. These studies showed that FXII/ $\mathrm{Zn}^{2+}$ promotes histone citrullination (Figure 9, $\mathrm{E}$ and $\mathrm{F}$; see complete unedited blots in the supplemental material). We stimulated neutrophils with FXII/ $\mathrm{Zn}^{2+}$ for $15,30,60$, and 120 minutes and determined that significant histone citrullination occurred as early as 30 minutes after FXII/Zn ${ }^{2+}$ stimulation (Figure $9, \mathrm{G}$ and $\mathrm{H}$ ), peaked at 60 minutes, and remained elevated at 120 minutes (Figure 9, G and H; ee complete unedited blots in the supplemental material). To better study the kinetics of NET formation compared with other agonists, we used a plate reader assay that detects extracellular DNA release with a cell-impermeable, extracellular DNA dye, SYTOX Green. fMLP and A23187 predictably caused rapid NETosis that became significant compared with media at 120 minutes and 130 minutes, respectively (Figure 9I). NETosis induced by PMA and FXII/ $\mathrm{Zn}^{2+}$ followed distinct kinetics and was slower (Figure 9I). FXII/ $\mathrm{Zn}^{2+}$, but not $\mathrm{Zn}^{2+}$ alone, induced significant release of extracellular DNA at 150 minutes, while PMA-mediated NET release occurred at 220 minutes (Figure 9I). When WT neutrophils were pretreated with the specific Akt2 inhibitor (Akti XII) before stimulation with FXII $/ \mathrm{Zn}^{2+}$, NETosis was significantly reduced compared with what occurred with FXII/ $\mathrm{Zn}^{2+}$ treatment (Figure 9I). Notably, when neutrophils were pretreated with LRG20, a domain II uPAR peptide that blocks FXII-uPAR interaction (22), pAkt2S ${ }^{474}$ was also significantly reduced (Supplemental Figure 9; see complete unedited blots in the supplemental material) and extracellular DNA release in response to FXII/ $\mathrm{Zn}^{2+}$ stimulation was suppressed (Figure 9I). These combined studies supported the assessment that the FXII-uPAR interaction 
A

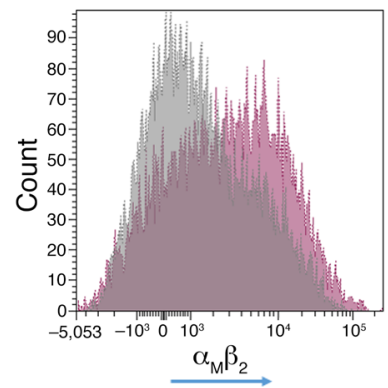

D

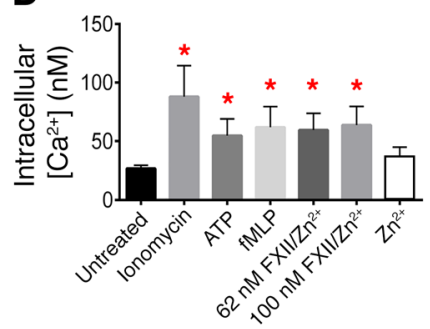

B

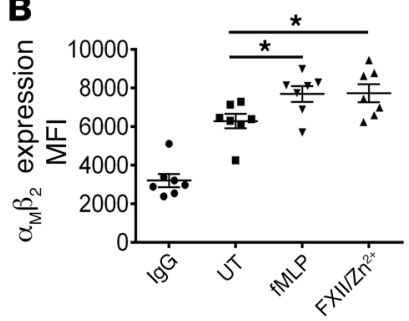

$\mathbf{E}$

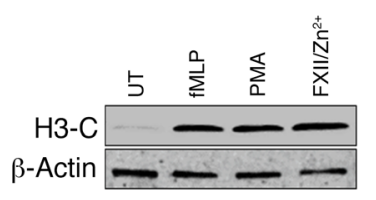

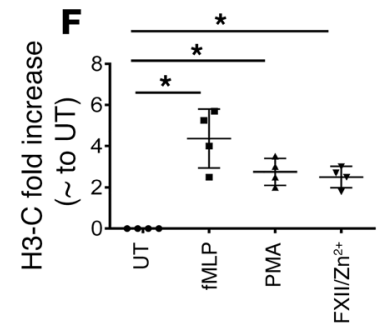

G
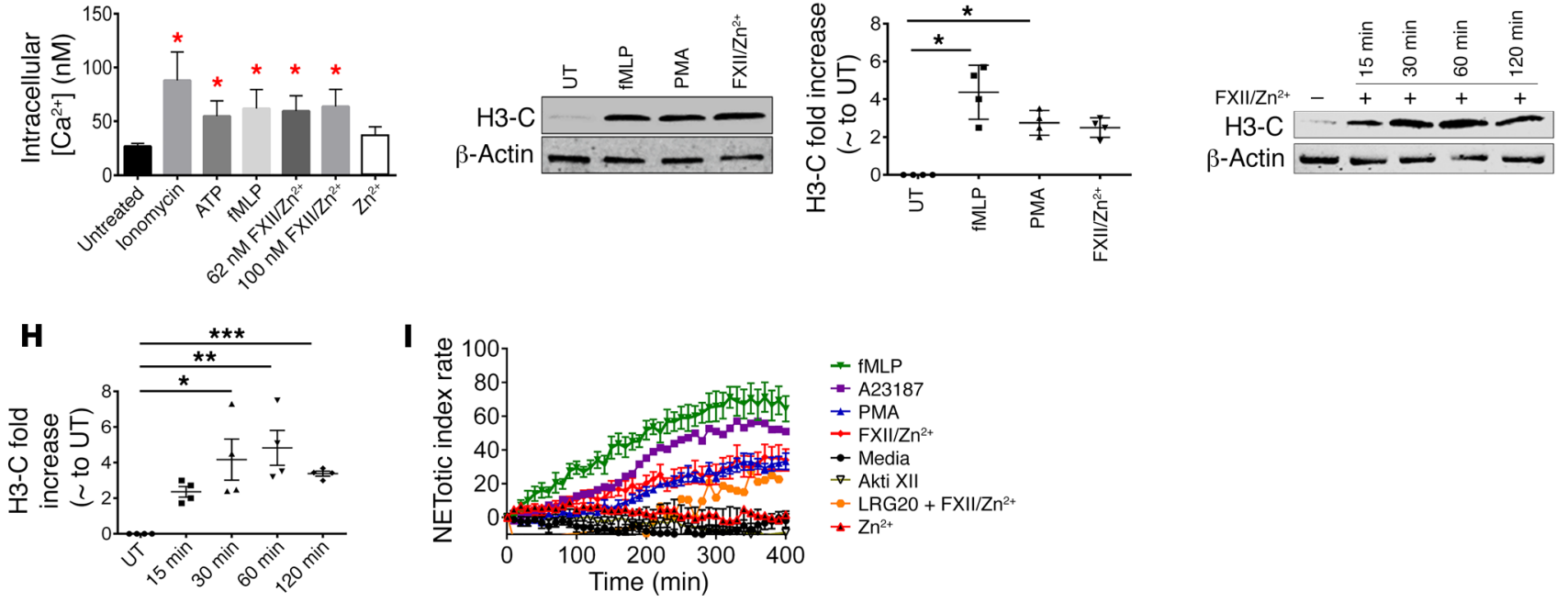

I

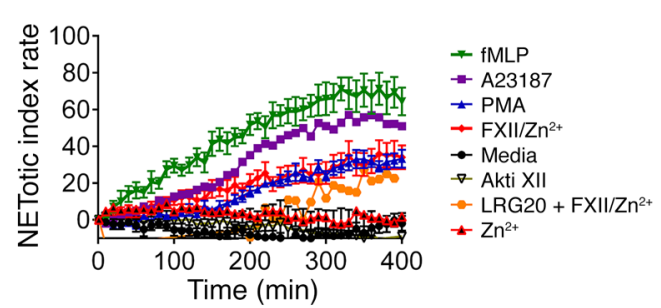

Figure 9. FXII signaling in neutrophils regulates $\alpha_{M} \beta_{2}$ surface expression, intracellular Ca ${ }^{2+}$ mobilization, and NET formation. (A) Surface expression of $\alpha_{\mathrm{M}} \beta_{2}$ integrin on UT (grey curve) and FXII/Zn $\mathrm{n}^{2+}$ stimulated (pink curve) neutrophils. Representative flow diagram of $n=7$ individual experiments. (B) Quantitation of $\alpha_{M} \beta_{2}$ antibody binding (mean fluorescent intensity [MFI]). Mean \pm SEM, $n=7$ experiments. ${ }^{*} P<0.03$ UT vs. FXII/Zn $n^{2+}, 1-$ way ANOVA. (C) WT neutrophils were loaded with $1 \mu \mathrm{M}$ Fluro-4-AM Ca ${ }^{2+}$ dye for 45 minutes and then treated with or without 5 mM ATP, $3 \mu M$ ionomycin, $10 \mu M$ fMLP, FXII $(62 \mathrm{nM}$ or $100 \mathrm{nM}) / 10 \mu \mathrm{M} \mathrm{Zn^{2+ }}$, or $\mathrm{Zn}^{2+}$ alone. Intracellular $\mathrm{Ca}^{2+}$ mobilization was measured at 30-second intervals for 30 minutes. (D) Intracellular Ca ${ }^{2+}$ concentration with various agonists. Mean $\pm \mathrm{SEM}, n=4$, each run in triplicate. ${ }^{*} P<0.0001 \mathrm{vs}$. UT cells, 1 -way ANOVA. (E) WT neutrophils were stimulated with fMLP, $100 \mathrm{nM}$ PMA, or FXII/Zn ${ }^{2+}$ for 120 minutes, and immunoblot analysis was performed for H3-C. Representative blot of $n=4$. (F) H3-C fold increase compared with UT neutrophils. Mean $\pm \mathrm{SEM}, n=4 .{ }^{*} P<0.007$ UT vs. FXII/Zn ${ }^{2+}$, 1-way ANOVA. (G) WT neutrophils were stimulated with $62 \mathrm{nM}$ FXII/10 $\mu \mathrm{M} \mathrm{Zn}{ }^{2+}$ for the indicated times. Immunoblot analysis was performed for H3-C. Representative blot of $n=4$. (H) H3-C fold increase compared with UT neutrophils. Mean $\pm \mathrm{SEM}, n=4$. ${ }^{*} P=0.007$ at 30 minutes; ${ }^{*} P<0.002$ at 60 minutes; ${ }^{* *} P=0.03$ at 120 minutes vs. UT, 1 -way ANOVA. (I) NETotic index rate of WT neutrophils activated with $\mathrm{fMLP}, 4 \mu \mathrm{M} \mathrm{A23187,1 \mu \textrm {M } P M A}$, FXII/Zn ${ }^{2+}$, or $\mathrm{Zn}^{2+}$ alone. Where indicated, neutrophils were preincubated with $5 \mu \mathrm{M}$ Akti XII or $300 \mu \mathrm{M}$ LRG20 for 30 minutes before stimulation with FXII/ Zn ${ }^{2+}$. Curves represent mean \pm SEM except A23187 (shown as mean). $n=5-9$ each run in triplicate. ${ }^{*} P<0.0001 \mathrm{FXII} / \mathrm{Zn}^{2+}$ vs. media at $150-400$ minutes, 2 -way ANOVA.

in neutrophils occurred upstream of NET formation. In sum, our data showed that FXII/uPAR contributed to neutrophil functions by generating intracellular mediator pAkt2 that (a) promoted the membrane translocation of $\alpha_{\mathrm{m}} \beta_{2}$ integrin, (b) increased intracellular calcium concentration, and (c) mediated histone citrullination and extracellular DNA release. The sum of these activities contributes to cell adhesion, migration, and NETosis.

Neutrophil FXII is the major determinant of cell influx at sites of inflammation. Since FXII influenced neutrophil functions and neutrophil-derived FXII was distinct from hepatic-derived FXII, studies determined whether deficiency of neutrophil FXII alone altered wound healing by performing adoptive BM transplantation experiments. At 6 weeks, all transplanted mice had normal complete blood count (CBC) and differential counts (Supplemental Table 3). WT BM transplanted into KO hosts corrected the leukocyte migration defect on the TG assay (Figure 10A). Alternatively, KO BM transplanted into WT hosts produced a PEC migration defect (Figure 10A). On flow cytometry, there was an increased percentage of peritoneal neutrophils in $\mathrm{F} 12^{--}$mice that had received WT BM (Figure 10B). Likewise, when WT BM was transplanted into KO hosts, day 2 wounds showed increased NE and pAkt content compared with those in any mice that had received KO BM (Figure 10, C and D). The number of neutrophils recruited into day 2 wounds was similarly increased in any host that had received WT BM (Figure 10E). Reduced neutrophil infiltration with less NE in WT mice that had received KO BM accelerated the rate of wound closure, similar to that seen in $\mathrm{F}^{2-/}$ (KO) mice (Figure $10, F$ and $G$ ). In contrast, the majority of day 5 wounds from KO animals that had received WT BM (WT to KO) remained open compared with wounds of KO mice that had received KO BM (Figure 10, $\mathrm{F}$ and $\mathrm{G})$. Based on our previous studies showing that $F 12^{-/-}$ mice exhibited reduced angiogenesis in skin wounds (22), we next determined whether the reduced neutrophil infiltration correlated with reduced CD31 (endothelial cell marker) expression. Day 5 


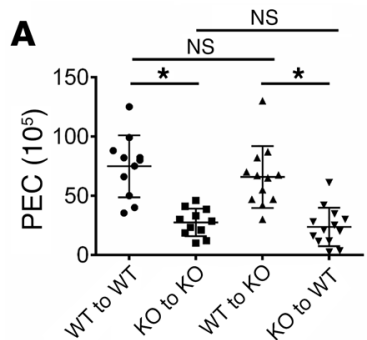

C

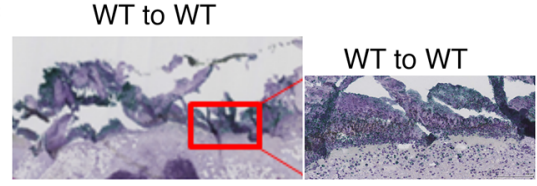

WT to KO

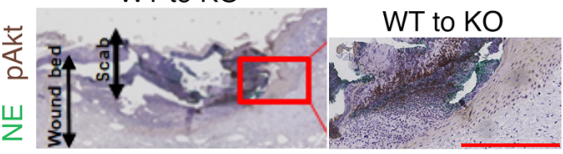

D $\mathrm{KO}$ to $\mathrm{KO}$

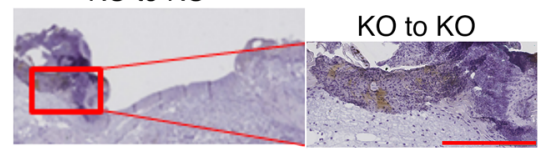

$\mathrm{KO}$ to WT

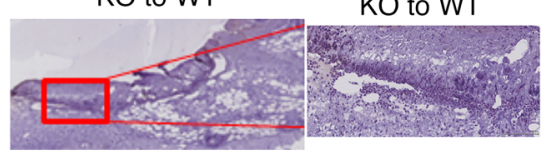

E

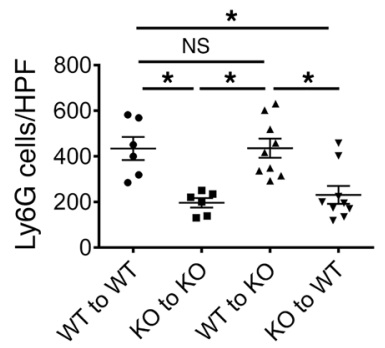

$\mathbf{F}$

WT to WT

$\mathrm{KO}$ to $\mathrm{KO}$

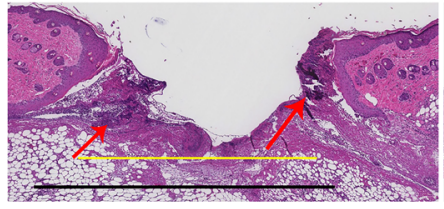

WT to KO

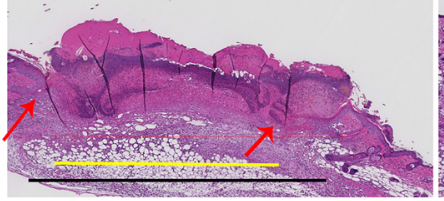

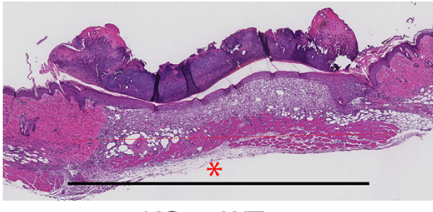

KO to WT

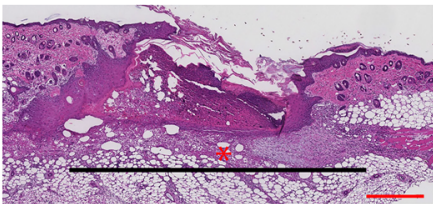

G

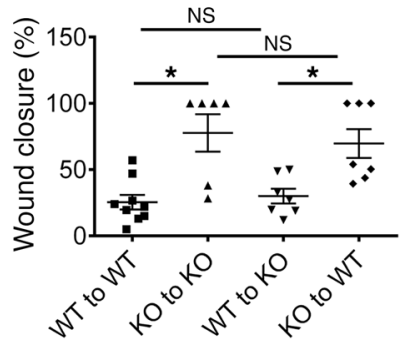

Figure 10. The influence of BM transplantation on inflammation and wound healing. (A) PEC number after TG-induced peritonitis in WT or KO (F12 $\left.{ }^{-1-}\right)$ hosts after WT or KO BM transplantation ( $n=11-13$ transplants/condition). Mean $\pm \mathrm{SEM} .{ }^{*} P<0.0004$ by 1-way ANOVA. (B) Flow cytometry of murine peritoneal lavage fluid 4 hours following TG-induced peritonitis in WT or KO BM-transplanted hosts. PEC were labeled with PE-conjugated anti-CD11b and PerCPCy 5.5-conjugated anti-F4-80 antibodies. Circles indicate the neutrophil subpopulation (CD11b-positive, F4-80-negative cells). The figure shows a representative flow cytogram of $n=4$ transplanted animals in each group. (C) NE and pAkt coimmunostaining from day 2 wounds of WT and (D) day 2 wounds of $F 12^{-1-}$ BM chimeras. Representative images of $n=4$ wounds in each group. Left panels in $\mathbf{C}$ and $\mathbf{D}$. Scale bars: $50 \mu \mathrm{m}$ (left panels); $100 \mu \mathrm{m}$ (right panels). Original magnification, $\times 4$ (left panels); $\times 20$ (right panels). (E) Number of Ly6G cells/high-power field in day 2 wounds of WT and F12 ${ }^{-/-}$chimeras. $n=6-9$ transplants/condition. Mean \pm SEM. ${ }^{*} P<0.007$, 1-way ANOVA. (F) H\&E-stained sections in day 5 wounds of WT and $F 12^{-/-} B M$ chimeras. Representative images of $n=6-9$ wounds in each group of transplanted animals. Original magnification, $\times 4$. Black lines demarcate the total length of original wound; yellow line represents the remaining wound gap. Red arrows represent the ingrowing epithelial tongues, closing wound. In photographs in which a red asterisk is seen, the wound gap is 0 , indicating that the wound has completely reepithelialized. Scale bars: $100 \mu \mathrm{m}$. Histologic sections were obtained using a Leica SCN 400 slide scanner equipped with a Hamamatsu line sensor color camera. Analysis was performed using Image software. (C) Wound closure in WT and $F 12^{-1-}$ BM chimeras, $n=6-9$ wounds/group. Mean $\pm S E M .{ }^{*} P=0.0004$, 1-way ANOVA.

wounds from all 4 groups of transplanted mice were examined for CD31 content (Supplemental Figure 10). We found that the area of CD31 staining (PECAM) was similar and not statistically different among KO mice that had received either WT or KO BM $(P=0.1$; Supplemental Figure 10, A and B). Accordingly, WT mice that had received either WT or KO BM had similar degrees of neoangiogenesis between them. However, neoangiogenesis was significantly greater in WT hosts than in FXII KO hosts $(P=0.0014)$ regardless of transplanted BM. These data indicate that wound neoangiogenesis is dependent upon the host, whereas the degree of neutrophil infiltration was dependent upon the source of BM. These combined data also show that (a) the effect of FXII on neutrophils is a $\mathrm{BM}$-endowed function and (b) the degree of inflammatory compo- nent of wounds is dependent upon neutrophil FXII and is the main determinant of wound healing. This latter observation has been recognized in human wounds as well $(48,49)$.

\section{Discussion}

The recognition in recent years that inflammatory processes and wound behavior are closely linked has led to a paradigm shift in our understanding of the molecular and cellular events that translate into wound healing. Excessive neutrophil activity or the persistence of neutrophils at wound sites contributes to the development of chronic, nonhealing wounds (50-53). Therefore, identifying the mediators that promote neutrophil priming will provide insight into how they can be inhibited or manipulated. We have 


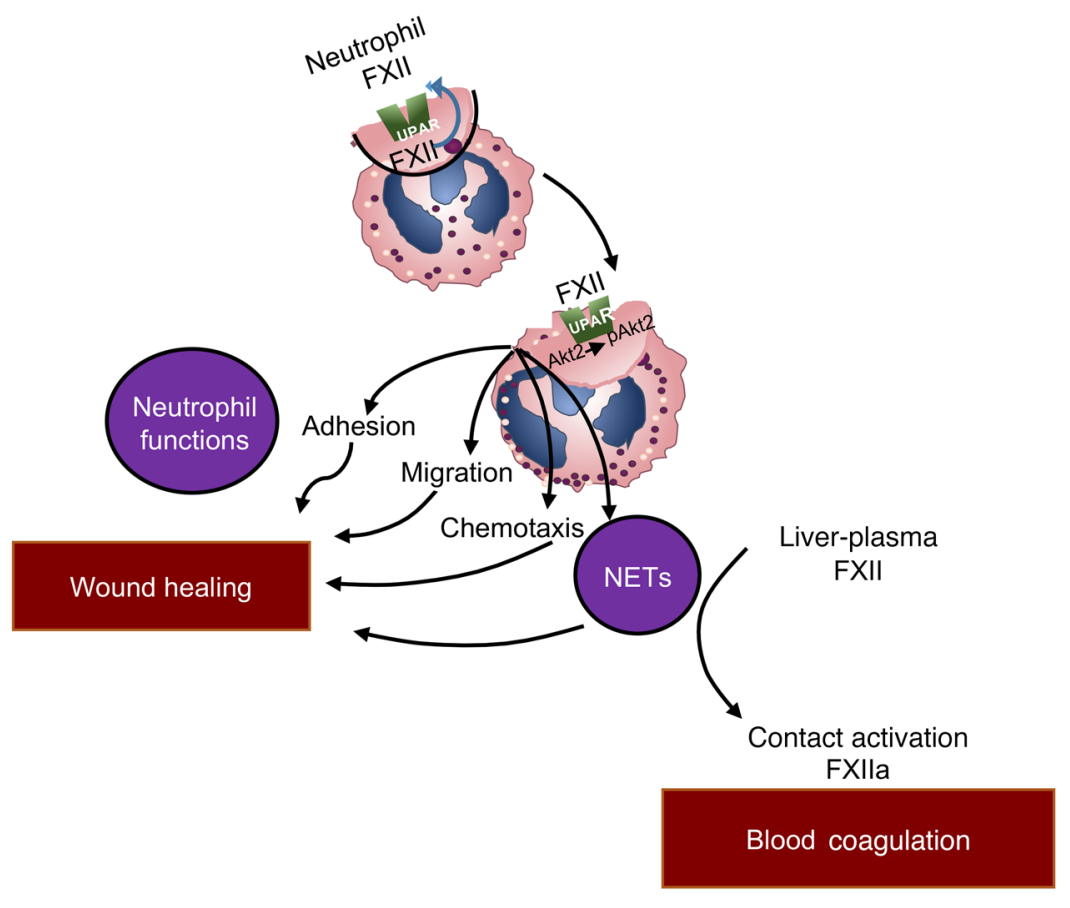

Figure 11. Role of FXII in inflammation and wound healing. Neutrophil FXII functions as an autocrine messenger upstream of plasma FXII. FXII signals through UPAR to promote $\mathrm{AktS}^{473}$ and $\mathrm{Akt} 2 \mathrm{~S}^{474}$ phosphorylation. Propagation of FXII-mediated neutrophil activities includes adhesion, migration, chemotaxis that leads to neutrophil trafficking at sites of inflammation, and NET formation. Activities of neutrophil-derived FXII are distinct from the function of plasma-derived FXII, which mediates contact activation on the surface of preformed NETs to produce FXIla for blood coagulation. found the FXII/uPAR/pAkt2 axis in neutrophils, which promotes cell activation and leads to impaired wound healing.

In the present study, we found that $F 12^{-/-}$mice exhibited decreased neutrophil recruitment in 2 models of sterile inflammation. When we measured the skin wound area daily in $\mathrm{F} 12^{-1}$ mice, we observed the maximum decrease between days 1-3 after wounding; this is the period where neutrophil recruitment overlaps with keratinocyte proliferation and matrix production. It has previously been reported that depletion of neutrophils accelerates the rate of reepithelialization in sterile nondiabetic and diabetic wounds (54). We therefore rationalized that the presence of neutrophils in wounds interferes with wound closure and that the decreased neutrophil migration in $\mathrm{F} 12^{-/-}$wounds accounts for their improved wound-healing potential. Targeting FXII production in the liver with a siRNA knockdown strategy unexpectedly does not affect neutrophil migration. Further, reconstituting plasma of $\mathrm{F}^{-/-}$mice with FXII does not correct the neutrophil migration defect. The finding that hepatic FXII does not influence neutrophil function has similarly been reported in Smad3-null monocytes in which plasma replacement with exogenous TFG- $\beta 1$ before wounding does not increase the number of monocytes recruited at wound sites (55).

We found F12 mRNA in murine neutrophils and FXII protein in murine and human peripheral neutrophils. When neutrophils are activated with fMLP, FXII antigen translocates to the plasma membrane and is secreted. Surface plasmon resonance studies confirmed that autocrine FXII interacts with UPAR on the neutrophil surface. Prior evidence of local FXII production has been shown in human lung fibroblasts from patients with acute respiratory distress syndrome (56).

We asked whether FXII signals in neutrophils. We examined Akt signaling because neutrophil invasion is strongly dependent upon it and because FXII signals in endothelial cells through Akt $(57,58)$. We show that FXII induces pAktS ${ }^{473}$ in both WT and F12-/- cells. Specifically, we show that when WT and $\mathrm{F} 12^{-/-}$neutrophils are treated with FXII/ $\mathrm{Zn}^{2+}$, pAkt $2 \mathrm{~S}^{474}$, the only isoform in neutrophils that translocates to the leading edge of polarized cells, is upregulated. The specific Akt2 inhibitor, Akti XII, abrogates FXII-induced phosphorylation of Akt $2 \mathrm{~S}^{474}$. It might be assumed that FXII-mediated effects on neutrophils relate to FXIa formation and downstream thrombin-induced cytokine expression. FXIa was previously described to alter the migration of human neutrophils (59), and $\mathrm{FXI}^{-/-}$mice had reduced levels of cytokines in plasma when subjected to polymicrobial sepsis (60). However, there is a growing body of evidence suggesting that components of the contact activation system may regulate inflammatory responses irrespective of their role in thrombin generation (17, 61-65). Here, we show that active site-inactivated FXII variants themselves promote Akt $2 S^{474}$ phosphorylation independently of any proteolytic activity. These results show that FXII is able to directly influence innate immune functions independently of its downstream enzymatic activity.

Given that the plasma concentration of FXII is approximately $450 \mathrm{nM}$ and that FXII's action on neutrophils relates to its zymogen form, we asked why the FXII/uPAR axis does not operate constitutively. In vivo, circulating FXII is not constitutively bound to cells in the intravascular compartment; it binds only when the local free zinc ion concentration (i.e., not bound to albumin) rises significantly from physiologic plasma levels of approximately $20 \mathrm{nM}(66,67)$ to a $7-10 \mu \mathrm{M}$ range $(21)$. The source of extracellular zinc was previously shown to derive from activated cells (21). Zinc dependency for FXII has previously been reported for both its enzymatic $(68-70)$ and nonenzymatic $(21,71)$ activities. To this end, BiaCore studies confirmed that FXII did not interact with UPAR in the absence of zinc. Zinc has also been shown to be an intracellular second messenger in immune cells $(72,73)$. When neutrophils were pretreated with TPEN, a cell-permeable $\mathrm{Zn}^{2+}$ selective chelator, prior to stimulation with FXII $/ \mathrm{Zn}^{2+}$, no signifi- 
cant Akt2S $\mathrm{S}^{474}$ phosphorylation was noted. These combined studies show that zinc supports FXII binding on neutrophils and is critical for FXII-mediated intracellular signaling.

The migration of neutrophils into tissues is a multistep process that involves tethering and rolling along the endothelium, chemokine-induced neutrophil adhesion, and actin-dependent polarization and migration before final diapedesis out of blood vessels (74). We determined that FXII/uPAR/pAkt2 upregulates the surface expression of $\alpha_{M} \beta_{2}$ integrin (inside-out signaling), increases intracellular calcium mobilization, and promotes extracellular DNA release. The sum of these activities contributes to neutrophil cell adhesion, migration, and NETosis. UPAR itself modulates integrin affinity and avidity (75-77). In endothelial cells, occupancy of UPAR by FXII promotes the formation of uPAR-integrin complexes that subsequently activate intracellular signaling pathways (outside-in signaling) through, at least, $\beta_{1}$ integrins (22). Whether the lateral association between FXII-uPAR and $\alpha_{\mathrm{M}} \beta_{2}$ integrin directly contributes to neutrophil functions is the focus of ongoing investigations. Finding a pathway for NET formation through FXII is therapeutically relevant, since current understanding is that their relationship lies solely on contact activation of circulating FXII on the surface of preformed NETs (10).

The observed phenotype of improved wound healing in $\mathrm{F} 12^{-1-}$ mice correlates with decreased appearance of neutrophils at sites of skin wound injury. Traditionally, neutrophils have been considered important for efficient wound repair. Besides clearing the initial rush of contaminating bacteria, neutrophils also are a source of proinflammatory cytokines that serve as some of the earliest signals to activate local fibroblasts and keratinocytes $(78,79)$. However, the value of having a large number of activated neutrophils in a healing wound has been recognized as a concern in recent years. Animal models show that excess neutrophil influx into wound sites impairs keratinocyte migration and proliferation (80). In addition, the persistence of neutrophils in wounds leads to unrestricted proteolytic activity mediated by neutrophil granular enzymes that are considered the final executor of a pathogenic chain leading to matrix disruption and proteolysis of growth factors and their receptors (49). Indeed, $\mathrm{NE}$ has previously been shown to be markedly increased in the exudate of nonhealing wounds and is thought to reflect a chronic, inflammatory, tissue-destructive microenvironment (48). In contrast, high levels of $\alpha 1$-antitrypsin, an in vivo NE inhibitor, are a biomarker of successful wound healing (49). NE associates with NETs and is critical to their function (81). Recent studies show that circulating neutrophils from diabetic humans are primed to produce NETs (51), and NETosis delayed diabetic wound healing in mice and humans (82). DNase 1, which disrupts NETs, accelerates wound healing in diabetic WT mice (51). Although efficient wound repair requires the coordinated effort of many different cell types (83), these data show that limiting the activity of neutrophils alone may be beneficial for the treatment of recalcitrant wounds.

It is not completely clear to us why FXII deficiency is beneficial in sterile inflammation models used in the present investigation. FXII-induced neutrophil activation may serve differential roles in nonsterile versus sterile inflammatory states. During infection(s), neutrophil responses constitute the first line of defense against pathogens. Prompt neutrophil recruitment and NET formation eventually lead to FXII contact activation and fibrin formation. The fibrin scaffold generated by this synergism entraps microbes within microvessels, limiting the systemic spread of infection while enhancing the clearance of pathogens by activated leukocytes $(84,85)$. In contrast with what occurs in the infectious setting, neutrophils can damage host tissues, contribute to the development of autoimmunity, and lead to a multitude of adverse outcomes $(82,86-88)$. In these latter scenarios, the contribution of FXII to neutrophil activation may be maladaptive and, among other outcomes, leads to persistent wound inflammation and impaired wound healing.

Taking these data together, we have identified the FXII/ uPAR/pAkt2 axis that regulates neutrophil functions. Our studies add to the current knowledge that neutrophils induce signals that amplify proinflammatory cellular responses. Specifically silencing FXII-mediated signaling in hematopoietic cells influences neutrophil adhesion, migration, and chemotaxis and results in improved wound healing. These FXII functions are independent of its enzymatic activity and upstream of NET formation (Figure 11). FXII signaling in neutrophils represents a heretofore unknown mechanistic link between neutrophil-driven inflammation and wound healing that should improve our understanding of the contribution of neutrophils in sterile inflammatory states and introduce a scientifically sound approach to targeting diseases that are characterized by sterile inflammation (23).

\section{Methods}

Animals. Six- to eight-week old male and female mice were used in equal numbers for all studies. $F 12^{-/-}$mice were provided by Francis J. Castellino (University of Notre Dame, South Bend, Indiana, USA) (24). Plaur $^{-1}$ mice were provided by Thomas Bugge (NIH, Bethesda, Maryland, USA) (89). The knockout mice were produced on a C57BL/6J background and backcrossed 7 generations. These animals were mated with WT mice (C57BL/6J, Jackson Laboratories) to make both heterozygous animals and rederived into $\mathrm{F}^{-2^{--}}$and $\mathrm{Plaur}^{-1}$. $\mathrm{F}^{-/-}$and Plaur $^{-/}$mice and littermate WT colonies were maintained by brother/sister mating. Every 10 generations, the $\mathrm{F}^{22^{-/}}$and $\mathrm{Plaur}^{-/}$ mice were mated with C57BL/6J to rederive knockouts from heterozygous mice. The genotyping of $F 12^{-/-}$mice was performed with oligonucleotide primer sets: a common reverse primer in exon 9 of the F12 gene (5'-GACGAAGCACCATGGACGTG, spanning bp 838-857 of the $F 12$ cDNA) and a forward primer in exon 8 of the $F 12$ gene (5'-GGCCACCACGCATTTTGCCG, spanning bp 801-820 of the F12 cDNA to detect the WT allele, $152 \mathrm{bp}$ ). Also, the same reverse primer and a forward primer residing within NEO (5'-GTAATACGACTCACTATAGG$\mathrm{GC})$ were used to detect the null allele (128 bp).

The WT Plaur allele was detected by PCR with the exon 3 primers (5' primer: 5'-GATGATAGAGAGCTGGAGGTGGTGAC-3') and Plaur reverse primer (5'-CACCGGGTCTGGGCCTGTTGCAGAGGT-3'), which generate a 148-bp product. The targeted Plaur allele was detected using an intron 3 primer (5'-TCATCAGTCCTCCCTGCTAAGGGC-3') and the hypoxanthine phosphoribosyl-transferase primer (5'-TATTAC-CAGTGAATCTTTGTCAGCAGTTCCC-3'), which generates a 272-bp PCR product.

Bdkrb2 $2^{-/}$mice, strain name B6/129S7-Bdkrb2 $2^{\text {tm1fh }}$, and their WT, B6129SF2/J mice (Bdkrb2--), originally were purchased from Jackson 
Laboratories, but then were mated to produce heterozygous animals from which WT and gene-deleted colonies were rederived.

Statistics. All data are presented as mean \pm SEM unless otherwise indicated. Differences between 2 groups were determined by unpaired 2-tailed Student's $t$ test. One-way ANOVA analysis with Bonferroni's correction was used to compare 3 or more related groups. Wound surface area over time was calculated with ordinary 2-way ANOVA. S-2302 hydrolysis is shown as a box plot with whiskers, representing minimum to maximum distribution. $P<0.05$ was considered significant.

Study approval. Animal care and procedures were reviewed and approved by the Institutional Animal Care and Use Committee at CWRU and performed in accordance with the guidelines of the American Association for Accreditation of Laboratory Animal Care and the NIH. All healthy donors and FXII-deficient patients enrolled in this study provided informed consent. The collection and use of blood samples for laboratory analysis were approved by the IRB of University Hospitals Cleveland Medical Center.

For more information, see Supplemental Methods.

\section{Author contributions}

EXS, CF, KLB, ATL, CN, EK, AG, ABM, MM, GRD, SI, AM, CCR, OA, LN, and GRD performed experiments. WMY, CKQ, and HJM provided assistance with $\mathrm{BM}$ transplantation and flow cytometry interpretation. EXS, CF, GRD, UAG, MTN, ASG, TR, and AHS conceptualized and planned experiments. EXS and AHS prepared the figures. EXS and AHS wrote the manuscript All authors reviewed the manuscript before submission.

\section{Acknowledgments}

The authors thank Jaehung Cho (University of Illinois College of Medicine) and Shigemi Matsuyama (CWRU School of Medicine) for expert advice on neutrophil signaling studies; Michael Sramkoski (CWRU Cancer Center Flow Cytometry Core Facility); Smarajit Bandyopadhyay (Cleveland Clinic) for assistance with BiaCore studies; Maryanne Pendergast (CWRU Neuroscience Imaging
Center); and Denise A. Hatala (CWRU) and Diane M. Mahovlic (Cleveland Clinic Lerner Research Institute Imaging Core) for expert histologic section preparation. This work was supported by the NIH, the National Heart, Lung, and Blood Institute (HL05277918, HL112666-2, HL126645-2, AI130131-1 to AHS), a Hemostasis and Thrombosis Research Society Mentored Research Award sponsored by Baxalta Inc., Healthcare Corp. (to EXS), an American Heart Association Scientist Development Award (to EXS), the Oscar D. Ratnoff Endowed Professorship (to EXS), the Young Scientist Foundation (to AG), an NIH-National Center for Research Resources Shared Instrumentation grant (1S10RR031845), the German Research Society (SFB877 TP A11 to TR), and a European Research Council grant (ERC-StG-2012-311575 F-12 to TR). The contents do not represent the views of the US Department of Veterans Affairs or the United States Government.

Address correspondence to: Evi X. Stavrou, 2143 WRB, 2103 Cornell Road, Cleveland, Ohio, 44106, USA. Phone: 216.368.6986; Email: evi.stavrou@case.edu.

CF's present address is: Department of Medicine, Beth Israel Deaconess Medical Center, Harvard Medical School, Boston, Massachusetts, USA.

CCR's present address is: Department of Pediatrics, University of California San Diego, San Diego, California, USA.

OA's present address is: Department of Medicine, MD Anderson Cancer Center, Houston, Texas, USA.

AG's present address is: DuPont Manual High School, Louisville, Kentucky, USA.

WMY and CKQ's present address is: AFLAC Cancer and Blood Disorders Center, Emory University, Atlanta, Georgia, USA.
1. Samuel M, Pixley RA, Villanueva MA, Colman RW, Villanueva GB. Human factor XII (Hageman factor) autoactivation by dextran sulfate. Circular dichroism, fluorescence, and ultraviolet difference spectroscopic studies. JBiol Chem. 1992;267(27):19691-19697.

2. Chen X, et al. Ordered adsorption of coagulation factor XII on negatively charged polymer surfaces probed by sum frequency generation vibrational spectroscopy. Anal Bioanal Chem. 2007;388(1):65-72.

3. Smith SA, Mutch NJ, Baskar D, Rohloff P, Docampo R, Morrissey JH. Polyphosphate modulates blood coagulation and fibrinolysis. Proc Natl Acad Sci U S A. 2006;103(4):903-908.

4. Müller F, et al. Platelet polyphosphates are proinflammatory and procoagulant mediators in vivo. Cell. 2009;139(6):1143-1156.

5. Smith SA, et al. Polyphosphate exerts differential effects on blood clotting, depending on polymer size. Blood. 2010;116(20):4353-4359.

6. Maas C, et al. Misfolded proteins activate factor XII in humans, leading to kallikrein formation without initiating coagulation. JClin Invest.
2008;118(9):3208-3218.

7. Kannemeier C, et al. Extracellular RNA constitutes a natural procoagulant cofactor in blood coagulation. Proc Natl Acad Sci U S A. 2007;104(15):6388-6393.

8. Gansler J, et al. Structural requirements for the procoagulant activity of nucleic acids. PLoS One. 2012;7(11):e50399.

9. van der Meijden PE, et al. Dual role of collagen in factor XII-dependent thrombus formation. Blood. 2009;114(4):881-890.

10. von Brühl ML, et al. Monocytes, neutrophils, and platelets cooperate to initiate and propagate venous thrombosis in mice in vivo. J Exp Med. 2012;209(4):819-835.

11. Nickel KF, et al. The polyphosphate-factor XII pathway drives coagulation in prostate cancer-associated thrombosis. Blood. 2015;126(11):1379-1389.

12. Renné T, et al. Defective thrombus formation in mice lacking coagulation factor XII. J Exp Med. 2005;202(2):271-281.

13. Kleinschnitz C, et al. Targeting coagulation factor XII provides protection from pathological throm- bosis in cerebral ischemia without interfering with hemostasis. JExp Med. 2006;203(3):513-518.

14. Pham M, Stoll G, Nieswandt B, Bendszus M, Kleinschnitz C. Blood coagulation factor XII--a neglected player in stroke pathophysiology. J Mol Med. 2012;90(2):119-126.

15. Matafonov A, et al. Factor XII inhibition reduces thrombus formation in a primate thrombosis model. Blood. 2014;123(11):1739-1746.

16. Rebuck JW. The skin window as a monitor of leukocytic functions in contact activation factor deficiencies in man. Am JClin Pathol. 1983;79(4):405-413.

17. Wachtfogel YT, et al. Purified plasma factor XIIa aggregates human neutrophils and causes degranulation. Blood.1986;67(6):1731-1737.

18. Henderson LM, Figueroa CD, Müller-Esterl W, Bhoola KD. Assembly of contact-phase factors on the surface of the human neutrophil membrane. Blood. 1994;84(2):474-482.

19. Chien P, Pixley RA, Stumpo LG, Colman RW, Schreiber AD. Modulation of the human monocyte binding site for monomeric immunoglobulin $\mathrm{G}$ by activated Hageman factor. J Clin Invest. 
1988;82(5):1554-1559.

20. Gordon EM, et al. Factor XII-induced mitogenesis is mediated via a distinct signal transduction pathway that activates a mitogen-activated protein kinase. Proc Natl Acad Sci U S A. 1996;93(5):2174-2179.

21. Mahdi F, Madar ZS, Figueroa CD, Schmaier AH. Factor XII interacts with the multiprotein assembly of urokinase plasminogen activator receptor, $\mathrm{gC} 1 \mathrm{qR}$, and cytokeratin 1 on endothelial cell membranes. Blood. 2002;99(10):3585-3596.

22. LaRusch GA, et al. Factor XII stimulates ERK1/2 and Akt through uPAR, integrins, and the EGFR to initiate angiogenesis. Blood. 2010;115(24):5111-5120.

23. Göbel K, et al. Blood coagulation factor XII drives adaptive immunity during neuroinflammation via CD87-mediated modulation of dendritic cells. Nat Commun. 2016;7:11626.

24. Iwaki T, Castellino FJ. Plasma levels of bradykinin are suppressed in factor XII-deficient mice. Thromb Haemost. 2006;95(6):1003-1010.

25. Iwaki T, Sandoval-Cooper MJ, Tenenhouse HS, Castellino FJ. A missense mutation in the sodium phosphate co-transporter Slc34a1 impairs phosphate homeostasis. J Am Soc Nephrol. 2008;19(9):1753-1762.

26. Gyetko MR, Sitrin RG, Fuller JA, Todd RF, Petty $\mathrm{H}$, Standiford TJ. Function of the urokinase receptor (CD87) in neutrophil chemotaxis. J Leukoc Biol. 1995;58(5):533-538.

27. Araújo RC, Kettritz R, Fichtner I, Paiva AC, Pesquero JB, Bader M. Altered neutrophil homeostasis in kinin B1 receptor-deficient mice. Biol Chem. 2001;382(1):91-95.

28. Austinat $\mathrm{M}$, et al. Blockade of bradykinin receptor $\mathrm{B} 1$ but not bradykinin receptor $\mathrm{B} 2$ provides protection from cerebral infarction and brain edema. Stroke. 2009;40(1):285-293.

29. Martin P. Wound healing--aiming for perfect skin regeneration. Science. 1997;276(5309):75-81.

30. Chen D, Hao H, Fu X, Han W. Insight into reepithelialization: How do mesenchymal stem cells perform? Stem Cells Int. 2016;2016:6120173.

31. Schmittgen TD, Zakrajsek BA, Mills AG, Gorn V, Singer MJ, Reed MW. Quantitative reverse transcription-polymerase chain reaction to study mRNA decay: comparison of endpoint and real-time methods. Anal Biochem. 2000;285(2):194-204.

32. May AE, Kanse SM, Lund LR, Gisler RH, Imhof BA, Preissner KT. Urokinase receptor (CD87) regulates leukocyte recruitment via beta 2 integrins in vivo. J Exp Med. 1998;188(6):1029-1037.

33. Simon DI, et al. Identification of a urokinase receptor-integrin interaction site. Promiscuous regulator of integrin function. J Biol Chem. 2000;275(14):10228-10234.

34. Degryse B, Resnati M, Czekay RP, Loskutoff DJ, Blasi F. Domain 2 of the urokinase receptor contains an integrin-interacting epitope with intrinsic signaling activity: generation of a new integrin inhibitor. J Biol Chem. 2005;280(26):24792-24803.

35. Sitrin RG, Todd RF, Albrecht E, Gyetko MR. The urokinase receptor (CD87) facilitates CD11b/ CD18-mediated adhesion of human monocytes. JClin Invest. 1996;97(8):1942-1951.

36. May AE, Neumann FJ, Schömig A, Preissner KT.
VLA-4 (alpha(4)beta(1)) engagement defines a novel activation pathway for beta(2) integrindependent leukocyte adhesion involving the urokinase receptor. Blood.2000;96(2):506-513.

37. Chen J, Tang H, Hay N, Xu J, Ye RD. Akt isoforms differentially regulate neutrophil functions. Blood.2010;115(21):4237-4246.

38. Stephens L, Ellson C, Hawkins P. Roles of PI3Ks in leukocyte chemotaxis and phagocytosis. Curr Opin Cell Biol. 2002;14(2):203-213.

39. Li J, et al. Neutrophil AKT2 regulates heterotypic cell-cell interactions during vascular inflammation. JClin Invest. 2014;124(4):1483-1496.

40. Kim K, Li J, Tseng A, Andrews RK, Cho J. NOX2 is critical for heterotypic neutrophil-platelet interactions during vascular inflammation. Blood. 2015;126(16):1952-1964.

41. Zhao Z, et al. Development of potent, allosteric dual Akt1 and Akt2 inhibitors with improved physical properties and cell activity. Bioorg Med Chem Lett. 2008;18(1):49-53.

42. Rizvi I, et al. Flow induces epithelialmesenchymal transition, cellular heterogeneity and biomarker modulation in 3D ovarian cancer nodules. Proc Natl Acad Sci US A. 2013;110(22):E1974-E1983.

43. Forsyth CB, Solovjov DA, Ugarova TP, Plow EF. Integrin alpha(M)beta(2)-mediated cell migration to fibrinogen and its recognition peptides. JExp Med. 2001;193(10):1123-1133.

44. Wang $\mathrm{Y}$, et al. Leukocyte engagement of platelet glycoprotein Ibalpha via the integrin Mac- 1 is critical for the biological response to vascular injury. Circulation. 2005;112(19):2993-3000.

45. Fuchs TA, et al. Novel cell death program leads to neutrophil extracellular traps. J Cell Biol. 2007;176(2):231-241.

46. Remijsen Q, et al. Neutrophil extracellular trap cell death requires both autophagy and superoxide generation. Cell Res. 2011;21(2):290-304.

47. Douda DN, Khan MA, Grasemann H, Palaniyar N. SK3 channel and mitochondrial ROS mediate NADPH oxidase-independent NETosis induced by calcium influx. Proc Natl Acad Sci U S A. 2015;112(9):2817-2822.

48. Eming SA, et al. Differential proteomic analysis distinguishes tissue repair biomarker signatures in wound exudates obtained from normal healing and chronic wounds. J Proteome Res. 2010;9(9):4758-4766.

49. Buchstein N, et al. Alternative proteolytic processing of hepatocyte growth factor during wound repair. Am J Pathol. 2009;174(6):2116-2128.

50. Roth Flach RJ, Czech MP. NETs and traps delay wound healing in diabetes. Trends Endocrinol Metab. 2015;26(9):451-452.

51. Wong SL, et al. Diabetes primes neutrophils to undergo NETosis, which impairs wound healing. Nat Med.2015;21(7):815-819.

52. Walker A, et al. Regulation of neutrophil apoptosis and removal of apoptotic cells. Curr Drug Targets Inflamm Allergy. 2005;4(4):447-454.

53. Grinnell F, Zhu M. Fibronectin degradation in chronic wounds depends on the relative levels of elastase, alpha1-proteinase inhibitor, and alpha2-macroglobulin. J Invest Dermatol. 1996;106(2):335-341.

54. Dovi JV, He LK, DiPietro LA. Accelerated wound closure in neutrophil-depleted mice. J Leukoc Biol. 2003;73(4):448-455.

55. Ashcroft GS, et al. Mice lacking Smad3 show accelerated wound healing and an impaired local inflammatory response. Nat Cell Biol. 1999;1(5):260-266.

56. Jablonska E, Markart P, Zakrzewicz D, Preissner KT, Wygrecka M. Transforming growth factor- $\beta 1$ induces expression of human coagulation factor XII via Smad3 and JNK signaling pathways in human lung fibroblasts. J Biol Chem. 2010;285(15):11638-11651.

57. Chin YR, Toker A. Function of Akt/PKB signaling to cell motility, invasion and the tumor stroma in cancer. Cell Signal. 2009;21(4):470-476.

58. Yang WL, Wu CY, Wu J, Lin HK. Regulation of Akt signaling activation by ubiquitination. Cell Cycle. 2010;9(3):487-497.

59. Itakura A, et al. Activated factor XI inhibits chemotaxis of polymorphonuclear leukocytes. J Leukoc Biol. 2011;90(5):923-927.

60. Bane CE, et al. Factor XI Deficiency alters the cytokine response and activation of contact proteases during polymicrobial sepsis in mice. PLOS One. 2016;11(4):e0152968.

61. Wachtfogel YT, et al. Human plasma kallikrein releases neutrophil elastase during blood coagulation. JClin Invest. 1983;72(5):1672-1677.

62. Toossi Z, Sedor JR, Mettler MA, Everson B, Young T, Ratnoff OD. Induction of expression of monocyte interleukin 1 by Hageman factor (factor XII). Proc Natl Acad Sci U S A. 1992;89(24):11969-11972.

63. Khan MM, et al. High-molecular-weight kininogen fragments stimulate the secretion of cytokines and chemokines through uPAR, Mac-1, and $\mathrm{gC1qR}$ in monocytes. Arterioscler Thromb Vasc Biol. 2006;26(10):2260-2266.

64. Stroo I, et al. Coagulation factor XI improves host defence during murine pneumonia-derived sepsis independent of factor XII activation. Thromb Haemost. 2017;117(8):1601-1614.

65. Hess R, et al. Coagulation factor XII regulates inflammatory responses in human lungs. Thromb Haemost. 2017;117(10):1896-1907.

66. Bal W, Sokołowska M, Kurowska E, Faller P. Binding of transition metal ions to albumin: sites, affinities and rates. Biochim Biophys Acta. 2013;1830(12):5444-5455.

67. Haase H, Hebel S, Engelhardt G, Rink L. The biochemical effects of extracellular $\mathrm{Zn}(2+)$ and other metal ions are severely affected by their speciation in cell culture media. Metallomics. 2015;7(1):102-111.

68. Björkqvist J, Lecher B, Maas C, Renné T. Zincdependent contact system activation induces vascular leakage and hypotension in rodents. Biol Chem. 2013;394(9):1195-1204.

69. Mutch NJ, Waters EK, Morrissey JH. Immobilized transition metal ions stimulate contact activation and drive factor XII-mediated coagulation. J Thromb Haemost. 2012;10(10):2108-2115.

70. MacQuarrie JL, et al. Histidine-rich glycoprotein binds factor XIIa with high affinity and inhibits contact-initiated coagulation. Blood. 2011;117(15):4134-4141.

71. Røjkaer R, Schousboe I. Partial identification of the $\mathrm{Zn}^{2+}$-binding sites in factor XII and its activation 
derivatives. Eur JBiochem. 1997;247(2):491-496.

72. Kitamura H, et al. Toll-like receptor-mediated regulation of zinc homeostasis influences dendritic cell function. Nat Immunol. 2006;7(9):971-977.

73. Yamasaki S, et al. Zinc is a novel intracellular second messenger. JCell Biol. 2007;177(4):637-645.

74. Phillipson M, Kubes P. The neutrophil in vascular inflammation. Nat Med. 2011;17(11):1381-1390.

75. Simon DI, et al. Mac-1 (CD11b/CD18) and the urokinase receptor (CD87) form a functional unit on monocytic cells. Blood. 1996;88(8):3185-3194.

76. Silvestri I, et al. Engaged urokinase receptors enhance tumor breast cell migration and invasion by upregulating alpha(v)beta5 vitronectin receptor cell surface expression. Int JCancer. 2002;102(6):562-571.

77. Gellert GC, Goldfarb RH, Kitson RP. Physical association of UPAR with the alphaV integrin on the surface of human NK cells. Biochem Biophys Res Commun. 2004;315(4):1025-1032.

78. Hübner G, Brauchle M, Smola H, Madlener M,
Fässler R, Werner S. Differential regulation of pro-inflammatory cytokines during wound healing in normal and glucocorticoid-treated mice. Cytokine. 1996;8(7):548-556.

79. Theilgaard-Mönch K, Knudsen S, Follin P, Borregaard $\mathrm{N}$. The transcriptional activation program of human neutrophils in skin lesions supports their important role in wound healing. JImmunol. 2004;172(12):7684-7693.

80. Mori R, Power KT, Wang CM, Martin P, Becker DL. Acute downregulation of connexin 43 at wound sites leads to a reduced inflammatory response, enhanced keratinocyte proliferation and wound fibroblast migration. JCell Sci. 2006;119(Pt 24):5193-5203.

81. Brinkmann V, et al. Neutrophil extracellular traps kill bacteria. Science. 2004;303(5663):1532-1535.

82. Fadini GP, et al. NETosis delays diabetic wound healing in mice and humans. Diabetes. 2016;65(4):1061-1071.

83. Reinke JM, Sorg H. Wound repair and regenera- tion. Eur Surg Res. 2012;49(1):35-43.

84. Engelmann B, Massberg S. Thrombosis as an intravascular effector of innate immunity. Nat Rev Immunol. 2013;13(1):34-45.

85. Luo D, et al. Fibrin facilitates both innate and $\mathrm{T}$ cell-mediated defense against Yersinia pestis. JImmunol. 2013;190(8):4149-4161.

86. Gupta S, Kaplan MJ. The role of neutrophils and NETosis in autoimmune and renal diseases. Nat Rev Nephrol. 2016;12(7):402-413.

87. Swirski FK, Nahrendorf M. Leukocyte behavior in atherosclerosis, myocardial infarction, and heart failure. Science. 2013;339(6116):161-166.

88. Demers M, Wagner DD. NETosis: a new factor in tumor progression and cancer-associated thrombosis. Semin Thromb Hemost. 2014;40(3):277-283.

89. Bugge TH, et al. Urokinase-type plasminogen activator is effective in fibrin clearance in the absence of its receptor or tissue-type plasminogen activator. Proc Natl Acad Sci U S A. 1996;93(12):5899-5904. 